This is the final peer-reviewed accepted manuscript of:

S. D'Adamo; S. Cetrullo; S. Guidotti; Y. Silvestri; M. Minguzzi; S. Santi; L. Cattini; G. Filardo; F. Flamigni; R. M. Borzì

Spermidine rescues the deregulated autophagic response to oxidative stress of osteoarthritic chondrocytes

Free Radical Biology \& Medicine 2020; 153: 159-172

The final published version is available online at:

http://dx.doi.org/10.1016/i.freeradbiomed.2020.03.029

Rights / License:

The terms and conditions for the reuse of this version of the manuscript are specified in the publishing policy. For all terms of use and more information see the publisher's website.

This item was downloaded from IRIS Università di Bologna (https://cris.unibo.it/)

When citing, please refer to the published version. 


\title{
Spermidine rescues the deregulated autophagic response to oxidative stress of osteoarthritic chondrocytes
}

\author{
Stefania D'Adamo ${ }^{\mathrm{a}, \mathrm{b}}$, Silvia Cetrullo ${ }^{\mathrm{a}}$, Serena Guidotti ${ }^{\mathrm{b}}$, Ylenia Silvestri ${ }^{\mathrm{a}}$, Manuela Minguzzi $^{\mathrm{b}}$, \\ Spartaco Santi ${ }^{\text {c,d }}$, Luca Cattini ${ }^{\mathrm{e}}$, Giuseppe Filardo ${ }^{\mathrm{f}}$, Flavio Flamigni ${ }^{\mathrm{a}}$, Rosa Maria Borzì e,* \\ a Dipartimento di Scienze Biomediche e Neuromotorie, Università di Bologna, Bologna, Italy \\ ${ }^{\mathrm{b}}$ Dipartimento di Scienze Mediche e Chirurgiche, Università di Bologna, Bologna, Italy \\ ${ }^{\mathrm{c}}$ Institute of Molecular Genetics, National Research Council (CNR), Bologna, Italy \\ d C/o IRCCS, Istituto Ortopedico Rizzoli, Bologna, Italy \\ e Laboratorio di Immunoreumatologia e Rigenerazione Tissutale, IRCCS Istituto Ortopedico Rizzoli, Bologna, Italy \\ f Applied and Translational Research Center, IRCCS Istituto Ortopedico Rizzoli, Bologna, Italy
}

\begin{abstract}
A B S T R A C T
Oxidative stress (OS) contributes to Osteoarthritis (OA) pathogenesis and its effects are worsened by the impairment of homeostatic mechanisms such as autophagy in OA chondrocytes. Rescue of an efficient autophagic flux could therefore reduce the bulk of damaged molecules, and at the same time improve cell function and viability. As a promising dietary or intra-articular supplement to rescue autophagy in OA chondrocytes, we tested spermidine (SPD), known to induce autophagy and to reduce OS in several other cellular models. Chondrocytes were obtained from OA cartilage and seeded at high-density to keep their differentiated phenotype. The damaging effects of OS and the chondroprotective activity of SPD were assessed by evaluating the extent of cell death, oxidative DNA damage and caspase 3 activation. The autophagy promoting activity of SPD was evaluated by assessing pivotal autophagic effectors, i.e. Beclin-1 (BECN-1), microtubule-associated protein 1 light chain 3 II (LC3-II) and p62. BECN-1 protein expression was significantly increased by SPD and reduced by $\mathrm{H}_{2} \mathrm{O}_{2}$ treatment. SPD also rescued the impaired autophagic flux consequent to $\mathrm{H}_{2} \mathrm{O}_{2}$ exposure by increasing mRNA and protein expression of LC3-II and p62. SPD induction of mitophagy was revealed by immunofluorescent co-localization of LC3-II and TOM20. The key protective role of autophagy was confirmed by the loss of SPD chondroprotection upon autophagy-related gene 5 (ATG5) silencing. Significant SPD tuning of the $\mathrm{H}_{2} \mathrm{O}_{2}$-dependent induction of degradative (MMP-13) inflammatory (iNOS, COX-2) and hypertrophy markers (RUNX2 and VEGF) was revealed by Real Time PCR and pointed at the SPD ability of reducing NF-KB activation through autophagy induction. Conversely, blockage of autophagy led to parallel increases of oxidative markers and p65 nuclear translocation. SPD also increased the proliferation of slow-proliferating primary cultures.Taken together, our findings highlight the chondroprotective, anti-oxidant and anti-inflammatory activity of SPD and suggest that the protection afforded by SPD against OS is exerted through the rescue of the autophagic flux.
\end{abstract}

\author{
Keywords \\ Osteoarthritis \\ Chondrocytes \\ Oxidative stress \\ Spermidine \\ Autophagy
}

\section{Introduction}

Osteoarthritis (OA) is the most common form of arthritis and one of the most significant causes of disability in the world, whose prevalence is strictly linked to aging [1].

At present, drug treatments of OA are unsatisfactory. Oxidative stress (OS) contributes to the OA pathogenesis [2]. Besides directly acting as a NF-kB activating trigger [3], OS can damage molecules and organelles and thus impacts on chondrocyte functions favouring catabo- lism over anabolism. A pivotal role in counteracting this derangement is played by cell homeostatic mechanisms such as autophagy [4].

Autophagy is a central housekeeping and a stress response system playing an essential quality control function with an anti-aging role by supporting the clearance of senescence-associated alterations of macromolecules and organelles. This process begins with the sequestration of intracellular organelles and proteins in autophagosomes that fuse with lysosomes in order to degrade the sequestered material.

However, autophagy is deregulated in OA chondrocytes that therefore cannot effectively dispose of damaged organelles. Much work

\footnotetext{
* Corresponding author. Via di Barbiano 1/10, 40136, Bologna, Italy.

E-mail addresses: stefania.dadamo2@unibo.it (S. D'Adamo); silvia.cetrullo@unibo.it (S. Cetrullo); sere.guidotti@gmail.com (S. Guidotti); ylenia.silvestri@gmail.com (Y. Silvestri);

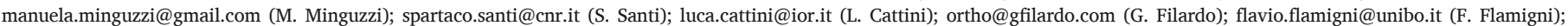
rosamaria.borzi@ior.it (R.M. Borzi)
} 
has been devoted to the search of a safe and effective mean to rescue autophagy. Recent research has suggested that the downregulated autophagy of OA compared to healthy chondrocytes derives from an altered kinetic pattern of the process as indicated by the different behavior of pivotal autophagic proteins after an oxidative challenge [5]. Rescue of an efficient autophagic flux could therefore at the same time improve clearance of damaged molecules, cell homeostasis and viability.

In this perspective, a safe opportunity may be represented by nutraceuticals and natural occurring compounds such as spermidine (SPD), an ubiquitous natural polyamine involved in a wide range of cellular processes, widely recognized to induce autophagy [6] and to reduce OS in several cellular models, as also recently reviewed $[7,8]$.

Recent reports have pointed at the ability of SPD to counteract disc degeneration via enhancement of autophagy in the cells of the nucleus pulposus [9] as well as its ability to reduce oxidative damage and inflammation in a model of cardiac infarction via enhancing the AMPK/ mTOR-mediated autophagic flux [10].

The object of this study has been to investigate the role of SPD in the context of OA cartilage, evaluating its ability to protect cultured human $\mathrm{OA}$ articular chondrocytes against hydrogen peroxide $\left(\mathrm{H}_{2} \mathrm{O}_{2}\right)$-induced OS by modulating autophagy through optimization of the autophagic flux. A previous paper had already investigated the autophagy promoting activity of SPD $[11,12]$. Sacitharan and coworkers indeed reported the aging-related decreased expression of autophagy related proteins (ULK1, BECN-1 and LC3) in both murine and human cartilage (even more decreased in OA cartilage), together with the SPD ability to rescue this expression and increase that of key chondrogenesis markers. Moreover, both murine and human aged cartilage showed reduced expression of the enzymes of the polyamine biosynthetic pathway. However, the in vitro study was carried out without the combined investigation of markers of cell damage and the use of proper controls [13] to correctly assess the autophagic flux. Moreover, in that study chondrocytes were not cultured at high density that helps in recovering the differentiated phenotype, according to published guidelines [14]. Hydrogen peroxide, together with peroxynitrite, is one of the major cartilage damaging ROS [15] and therefore our experimental model reproduces in vitro the OA environment. The DNA damaging activity of $\mathrm{H}_{2} \mathrm{O}_{2}$ evokes stress induced homeostatic responses ordered through a range of increasing severity from pro-survival responses (cell cycle arrest, DNA repair, and autophagy) to pro-elimination response (differentiation, senescence and apoptosis) [16]. Chondrocyte susceptibility to OS markedly changes as a function of the culture density and exposure length [17]. Compared to a previous report [5] we chose an $\mathrm{H}_{2} \mathrm{O}_{2}$ exposure (dose/time) beyond the threshold of cell death to fully assess SPD chondroprotective activity. Exposure to OS is responsible for multiple cell features such as oxidative damage to mitochondria and genomic DNA. OS damaged mitochondria then lose their fundamental anti-aging activity and become instead promoter of cellular senescence, chronic inflammation and age-dependent cell dysfunction [18,19]. In these conditions, an essential homeostatic role is played by correct coupling of mitophagy (i.e. autophagy and clearance of the damaged mitochondria) and mitochondrial biogenesis [20]. However, if this fails, a persistent inflammatory activation occurs, with OS dependent chronic NF- $\kappa$ B activation [3]. At the genomic DNA level, OS causes double strand breaks tagged by $\gamma \mathrm{H} 2 \mathrm{AX}$, a phosphorylated histone marker that indicates foci where the cell organizes the DNA damage response in order to repair the damage. However, the persistence of $\gamma \mathrm{H} 2 \mathrm{AX}$ is itself a NF-KB stimulus [21] and a senescence promoting trigger [22]. Cells that are unable to get rid of OS damage then accumulate damaged molecules and organelles and become dysfunctional and unable to proliferate (i.e. senescent). Their persistent and sustained NF-kB activation is then responsible for the release of a wide range of inflammatory cytokines and matrix degrading enzymes that collectively constitute the Senescence Associated Secretory Phenotype (SASP) [23] and that affect the bystander cells and tissues. Recent research has provided the proof of concept that selectively targeting senescent cells and their SASP in OA by a new class of therapeutics (i.e. the "senolytics") has the potential to affect the natural course of the disease [24]. Another possibility is represented by "senomorphic" strategies, i.e. the use of drugs or food derived components that enhance homeostatic/pro-survival responses in the cells, such as autophagy, thus rescuing senescent cells from their dysfunctional status and attenuating NF- $\mathrm{\kappa B}$ signaling. Based on previous information, SPD is a suitable senomorphic candidate [25]. Senescence is a key driver of OA [26] and a major OA pathogenetic mechanism to target with potentiation of autophagy [27].

Autophagy itself is a homeostatic response triggered by OS. Despite recent work has warned that autophagy might play both anti-senescence and pro-senescence roles, in a context specific manner $[28,29]$, in most cases autophagy plays cell protective and anti-inflammatory effects, provided the functionality of downstream autophagic flux. Indeed, when the latter is impaired, increased autophagic vacuolization indicates decreased, rather than increased, autophagy. When evaluating candidate autophagy modulators, it is therefore important to couple autophagy assessment with evaluation of their effects on cell health.

Therefore, together with assessment of autophagy markers [BECN-1, the microtubule-associated protein 1 light chain 3 II (LC3-II) and p62, markers of autophagosome and of increased autophagic flux, respectively [13]] we evaluated the effect on cell damage $(\gamma \mathrm{H} 2 \mathrm{AX}$ foci, 8-oxo-dG adducts, caspase 3 activation and localization) and cell death.

\section{Materials and methods}

\subsection{Chondrocyte primary cultures}

The study was conducted in accordance with the 1975 Declaration of Helsinki; informed consent was obtained from all patients before surgery and the protocol was approved by the Ethics Committee of Istituto Ortopedico Rizzoli (SPD-OMEO, Prot.gen.n.ro 0019715, September 28,2016$)$. The patients admitted to the study had to meet inclusion and exclusion criteria (the latter excluded patients with BMI higher than 35, rheumatic diseases, diabetes, severe chronic infective diseases or malignancies, severe psychiatric diseases or use of steroid drugs). Chondrocytes were obtained from the cartilage of tibial plateau and femoral condyles of 31 OA patients ( 23 female, 8 male, mean \pm SD age: $70.1 \pm 5.7$, mean \pm SD Kellgren-Lawrence grade: $3.5 \pm 0.5$ ) that had underwent knee arthroplasty. Written informed consent was collected from all individuals involved in the study.

\subsection{Culture and treatment of chondrocyte primary cultures}

Chondrocytes were isolated by sequential enzymatic digestion, expanded in vitro up to passage p1, cultured and seeded in 10\% FCS-DMEM [30] in 12 well plates at high-density ( 250.000 cells/well, i.e. 62.500/ $\mathrm{cm}^{2}$ ) to keep the proper chondrogenic phenotype [17] and let for $36 \mathrm{~h}$ to restore cell-ECM interactions required for proper signaling of chondrocytes [31]. After $24 \mathrm{~h}$ starvation, the cells were pretreated with SPD (Sigma S2501, spermidine trihydrochloride), $100 \mathrm{nM}, 24 \mathrm{~h}$. The starvation step was useful to reduce the endogenous polyamine pool so to better highlight the effect of $100 \mathrm{nM}$ exogenous SPD pre-treatment. This subumolar dose was selected on the basis of previous literature hints that indicate a narrow useful dosage window in which addition of exogenous SPD may support cell homeostatic activities by increasing the unbound pool without exerting inflammation or apoptosis promoting effects [32]. Previous work from our group showed that 1-10 $\mu$ molar doses instead promote chondrocyte terminal differentiation [33], in keeping with previous observations of high SPD levels in the extracellular matrix of the ossifying cartilage [34]. Moreover, preliminary experiments comparing SPD pre-treatment with 100, 500 
and $1000 \mathrm{nM}$ showed that the $100 \mathrm{nM}$ SPD pre-treatment was more effective and more reproducible in reducing cell death.

To test SPD ability to reduce cell death, OS was induced through an overnight incubation with $\mathrm{H}_{2} \mathrm{O}_{2}(500 \mu \mathrm{M}) . \mathrm{H}_{2} \mathrm{O}_{2}$ exposure time and concentration were chosen on the basis of previous data that pointed at an increased resistance to OS of chondrocytes plated at high density [17]. A shorter exposure $(4 \mathrm{~h})$ was used to investigate effects on protein and RNA expression.

After $\mathrm{H}_{2} \mathrm{O}_{2}$ exposure, several different experimental procedures were undertaken, as described thereafter, to collect integrated information to better understand SPD effects on the cells. Some cultures were committed to the assessment of cell viability and $\gamma \mathrm{H} 2 \mathrm{AX}$ by flow cytometry, some other cultures underwent either protein or RNA extraction and committed to either Western blot analysis of key autophagic proteins or to caspase-3 activity assessment, or to Real Time RT-PCR assessment of effects on gene transcription. Some other cultures were used for immunofluorescence experiments. While in the first two cases, at the end of the experimental protocol the cells were recovered by trypsinization and treated according to their subsequent use, for immunofluorescence the cells were initially seeded in 8 well chamber slides (Millipore), fixed with $2 \%$ paraformaldehyde (PFA) 10 ' at the end of the treatments and kept in PBS until the time of processing.

\subsection{Evaluation of cell viability and markers of OS}

The extent of cell death was therefore analyzed by Flow Cytometric detection of Sytox Green staining, a non toxic probe that rapidly penetrates into dead/dying cells unable to keep membrane selective permeability, while it is excluded by viable cells [35]. This probe allows a clear distinction between viable and dying/dead cells with medians separated by two log fluorescence. To this end, 5 different primary cultures were treated as indicated above, and at the same time of $\mathrm{H}_{2} \mathrm{O}_{2}$ delivery, supplemented with sub-cytotoxic (1:1000) amount of Sytox Green (SYTOX ${ }^{\mathrm{TM}}$ Green Dead Cell Stain, for flow cytometry, Thermofisher) staining. Cell were collected by trypsinization and subjected to flow cytometric analysis. The remaining cells were fixed with $2 \%$ PFA for $10 \mathrm{~min}$ and kept for further analysis as indicated below. Results were expressed as percentage of dead cells.

The effects of OS exposure on chondrocytes and the extent of SPD cytoprotection were evaluated exploiting multiple markers of oxidative cell damage in either plated cells or cells recovered after treatment.

At first, SPD cytoprotection from OS was measured by flow cytometric analysis of reduction of $\gamma \mathrm{H} 2 \mathrm{AX}$, markers of double strand breaks. To this end, a flow cytometric analysis was undertaken, essentially as described in Ref. [36]. For each sample, the Mean Channel of Fluorescence Intensity increment (MCFI) was recorded, i.e. the net fluorescence increase of the sample stained with the antibody over the corresponding sample treated with isotype control antibody.

8-oxo-dG, a known marker of oxidative damage to DNA was evaluated by immunofluorescence (Anti-8-oxo-dG Monoclonal Antibody (clone 2E2), Trevigen \# 4354-MC-050) exploiting colocalization with active caspase 3, as detailed below. Activation of the effector caspase 3/ 7 downstream mitochondrial oxidative damage [37] was also assessed using protein cell lysates obtained as described thereafter for Western blot and measuring the cleavage of the fluorogenic peptide substrate Ac-Asp-Glu-Val-Asp-7-amido-4-methylcoumarin (Ac-DEVD-AMC) by evaluating the accumulation of a fluorescent product as described in Ref. [36]. The level of caspase activity was therefore measured as fluorescence intensity, normalized per mg protein, and expressed as fold increase the untreated controls. Moreover, the pattern of activation was also investigated with immunofluorescence (as detailed below), to assess the differential intracellular localization across the different conditions.

\subsection{Evaluation of autophagy}

Assessment of autophagy was carried out by evaluating the modulation of key autophagic proteins by Western blot essentially as previously described [38]. Equal amounts $(20 \mu \mathrm{g})$ of cell extracts were subjected to electrophoresis in $15 \%$ gels, blotted onto nitrocellulose membranes and probed with primary antibody at $4{ }^{\circ} \mathrm{C}$ overnight. The following proteins were investigated: 1) BECN-1 (Cell Signaling Technology Rabbit anti- Beclin-1, Pab\# 3738), a protein that acts during the initiation stage of autophagy by forming the isolation membrane, a double-membrane structure that engulfs cytoplasmic material to start forming the autophagosome; 2) LC3A/B (Cell Signaling Technology, Rabbit anti- LC3A/B Pab\#4108), involved in the formation of autophagosome, through the appearance of a membrane-associated, phosphatidylethanolamine (PE)-conjugated form of LC3 (LC3-II), detectable by Western blot, indicator of autophagosome formation and 3) the LC3-binding protein SQSTM1/p62 (Santa Cruz Biotechnology, mouse monoclonal \#SC28359), critical for autophagic degradation of various cargos and itself undergoing selective turnover through autophagy. $\beta$-actin (mouse monoclonal antibody \# 2228, Sigma-Aldrich) served as loading control and for normalization of band intensity.

After washes, membranes were incubated with horseradish peroxidase-conjugated anti-rabbit (Cell Signaling Technology) or anti-mouse (Santa Cruz Biotechnology) IgG for $1 \mathrm{~h}$. The chemiluminescent signals were detected using an ECL system (Luminata ${ }^{\mathrm{TM}}$ Crescendo, Millipore). Signals were detected with a ChemiDoc apparatus. The combined evaluation of LC3 and p62 immunoblotting was undertaken following the guidelines detailed by Ref. [13] for a correct evaluation of autophagy and for the assessment of the autophagic flux. In details, samples were assessed both in the absence and in the presence of $50 \mu \mathrm{M}$ chloroquine (CQ), a lysosomal protease inhibitor.

Finally, occurrence of mitophagy (selective degradation of mitochondria by autophagy) was analyzed by assessing the immunofluorescent colocalization of LC3B-II and TOM20, a mitochondrial outer membrane marker. The use of anti-LC3B antibody (Novus Biologicals \# LC3B/ MAP1LC3B rabbit polyclonal antibody NB100-2220) allowed us to stain the type of autophagosome found more abundant throughout the cytoplasm and less at the nuclear level, and therefore more likely corresponding to those in charge of disposing of damaged organelles and molecules, according to recent literature [39]. A quantitative colocalization analysis as described in Ref. [40] was carried out using the overlap coefficient $\mathrm{K} 1$, in order to compare the different culture conditions. 15 randomly selected cells for each condition were measured.

\subsection{Confirmation of the role of autophagy in the protective effects exerted by $S P D$}

A confirmation of the essential role of autophagy in sustaining the chondroprotective activity of SPD was carried out using Small interfering RNA-mediated gene silencing of ATG5 (ON-TARGET plus ATG5 siRNA) essentially as in Ref. [38] in 4 different primary cultures left untreated or treated with hydrogen peroxide with or without SPD pre-treatment. Results were compared with reference samples treated with control siRNA (ON-TARGET plus Non-targeting Pool, Dharmacon). Cell viability as estimated through the trypan blue exclusion test represented the readout of these treatments. Efficiency of the knockdown was assessed by Western blot detection of ATG5 (by the Cell Signaling Technology rabbit monoclonal antibody \#D5F5U) in siATG5 versus siCTRL treated cells. 
2.6. In situ analysis of correlated patterns: 8-oxo-dG and caspase 3 activation; LC3B and TOM20; BECN-1 and LC3

The correlated pattern of some selected markers was investigated by immunofluorescence exploiting double labeling, i.e. combination of two primary antibodies of mouse and rabbit origin, subsequently revealed with the combined use of anti-mouse and anti-rabbit secondary antibody linked with either Alexa Fluor 488 or Alexa Fluor 555. The combined analyses were as follows: 1) 8-oxo-dG (TREVIGEN 4354-MC-050, mouse monoclonal antibody, $2 \mu \mathrm{g} / \mathrm{ml}$ ) with active CP3 (R\&D AF835, rabbit polyclonal antibody, $5 \mu \mathrm{g} / \mathrm{ml}$ ) to investigate the effect of intracellular OS on CP3 activation; 2) LC3B (NOVUS BIOLOGICALS \# NB100-2220, rabbit polyclonal antibody, $5 \mu \mathrm{g} / \mathrm{ml}$ ) with TOM20 (SCBT sc-17764, mouse monoclonal antibody, $5 \mu \mathrm{g} / \mathrm{ml}$ ) to assess mitophagy; 3) LC3B with BECN-1 (BD612112, mouse monoclonal antibody, $5 \mu \mathrm{g} /$ $\mathrm{ml}$ ) to investigate the correlated behaviour of these two autophagy related proteins during cell division.

After the treatments, chondrocytes were washed with PBS and fixed with $2 \%$ PFA for $30^{\prime}$ at RT. At the time of performing the immunofluorescence experiments, a step of post-fixation was carried out with $90 \%$ methanol for $10^{\prime}$ on ice, as recommended to get rid of free LC3B proteins not organized as autophagosome. The samples were pretreated for antigen unmasking with $0.02 \mathrm{U} / \mathrm{ml}$ Chondroitinase ABC (SIGMA) in $50 \mathrm{mM}$ Tris/ $\mathrm{HCl} \mathrm{pH} 8.0$ for $20^{\prime}$ at $37^{\circ} \mathrm{C}$. After extensive washes with $\mathrm{TBS}$, the non-specific bindings were blocked with a $5 \%$ bovine serum albumin (BSA), $5 \%$ Normal Donkey serum and $0.1 \%$ Triton in TBS for $30^{\prime}$ at RT, and washed again. The mouse and rabbit primary antibodies were delivered together at the concentration indicated above and left overnight in humid chamber at $4{ }^{\circ} \mathrm{C}$. After rinsing in TBS, the signals were revealed by a $15 \mu \mathrm{g} / \mathrm{ml}$ Alexa Fluor ${ }^{\circledR} 555$ donkey anti-rabbit/mouse and a $15 \mu \mathrm{g} / \mathrm{ml}$ Alexa Fluor ${ }^{\circledR} 488$ donkey anti-mouse/rabbit IgG secondary antibody conjugates (Novex), incubated 30' at RT together with $1 \mu \mathrm{g} / \mathrm{ml}$ Hoechst 33342 (Sigma Aldrich) for nuclear counterstaining. At the end, the samples were mounted with the addition of anti-fading (1\% 1,4 Diazobicyclo (2.2.2) Octane (DABCO, SIGMA) in $90 \%$ glycerol, in $0.1 \mathrm{M} \mathrm{pH} 8.0$ Tris-HCl), sealed with nail-polish and stored refrigerated and in the dark for subsequent analysis. Pictures were taken at high magnification with a Nikon A1-R confocal laser scanning microscope equipped with either a Nikon $20 \times, 0.95$ NA objective lens or a Nikon $60 \times, 1.4$ NA objective lens, and with 405, 488 and $561 \mathrm{~nm}$ laser lines to excite DAPI (blue), Alexa 488 (green) and Alexa 555 (red) fluorescence signals, respectively. Emission signals were detected by a photomultiplier tube (DU4) preceded by emission filters BP 525/50 $\mathrm{nm}$ and BP 595/50 nm for Alexa Fluor 488 and Alexa Fluor 555, respectively. Laser scanning, image acquisition and processing were performed with Nikon Imaging Software NIS Elements AR-4 (Nikon Inc., USA). Optical sections were spaced $* 0.5 \mu \mathrm{m}$ along the z-axis and

Table 1

Details of primers used for gene expression analysis.

\begin{tabular}{|c|c|c|c|}
\hline GENE & Primer forward & Primer reverse & Amplicon Length \\
\hline GAPDH (transcript $V v 1-4,7$ ) & TGGTATCGTGGAAGGACTCA & GCAGGGATGATGTTCTGGA & 123 \\
\hline LC3B & AGCATCCAACCAAAATCCCG & TGAGCTGTAAGCGCCTTCTA & 144 \\
\hline P62 (transcript Vv 1-3) & AAGCCGGGTGGGAATGTTG & GCTTGGCCCTTCGGATTCT & 213 \\
\hline SOX9 & GAGCAGACGCACATCTC & CCTGGGATTGCCCCGA & 118 \\
\hline RUNX2 (transcript Vv 1,2,4,7) & GGAATGCCTCTGCTGTTATG & AGACGGTTATGGTCAAGGTG & 105 \\
\hline MMP13 & AGCCACTTTATGCTTCCTGA & TGGCATCAAGGGATAAGGAAG & 130 \\
\hline TIMP3 & CCTTGGCTCGGGCTCATC & GGATCACGATGTCGGAGTTG & 121 \\
\hline VEGF (transcript $V v$ 1-9) & TGATGATTCTGCCCTCCTC & GCCTTGCCTTGCTGCTC & 82 \\
\hline iNOS & ACATTGATCAGAAGCTGTCCCAC & AAAGGCTGTGAGTCCTGCAC & 235 \\
\hline COX2 & CAGCACTTCACGCATCAGTTT & GCGCAGTTTACGCTGTCTA & 129 \\
\hline
\end{tabular}

were digitized with a scanning mode format of $1024 \times 1024$ pixels and 4096 grey levels.

For intensity quantitative assessment, single sections passing through the nuclei were analyzed, and on each nucleus and cytoplasm, the fluorescence intensity of a round area (with a $5 \mu \mathrm{m}$ radius) roughly corresponding to half the nuclear area was measured.

\subsection{Evaluation of SPD effects on mRNA levels of selected genes}

The effects of cell treatments were also assessed at the level of mRNA expression, essentially as detailed in Ref. [38] by Real Time RT-PCR.

At first, modulation of key autophagic proteins was assessed by evaluating the gene expression of $L C 3 B$ and of $p 62$ (see Ref. [38]). Then, we explored the effects of the treatments on differentiation markers (SOX9 and RUNX2), degradative and angiogenetic markers pivotal in OA (MMP13 and its major counteractor, i.e. TIMP3, and VEGF) and on inflammatory markers (iNOS and COX2), suggestive of a NF- $\mathrm{kB}$ activating activity of hydrogen peroxide. The amount of mRNA was normalized for $G A P D H$ expression in each sample and referred to untreated, control sample.

The primers used are listed in Table 1.

\subsection{In situ analysis of the correlated pattern of 8-oxo-dG and NF- $\mathrm{NB}$ activation}

To further investigate the effects of autophagy inhibition on intracellular OS and transcription of NF- $\mathrm{KB}$ target genes, we performed an immunohistochemical evaluation of the level of 8-oxo-dG and nuclear localization of p65 (i.e. of the extent of NF- $\mathrm{KB}$ activation) in cells treated or not with CQ, a lysosomal protease inhibitor able to block the autophagic flux [13].

Immunofluorescence was carried out essentially as described above, by using the following primary antibodies: 8-oxo-dG (TREVIGEN, 4354-MC-050, $2 \mu \mathrm{g} / \mathrm{ml}$ ) and p65 (AbCAM AB7970, rabbit polyclonal antibody, $5 \mu \mathrm{g} / \mathrm{ml}$ ) that were revealed by $15 \mu \mathrm{g} / \mathrm{ml}$ Alexa Fluor 555 donkey anti-mouse and $15 \mu \mathrm{g} / \mathrm{ml}$ Alexa Fluor 488 donkey anti-rabbit IgG secondary antibody conjugates (Novex).

The effects on NF-kB activation (the change in the intensity of nuclear p65 staining) and the level of oxidative damage in the cytoplasm microscopy. Single sections passing through the nuclei were analyzed, and the fluorescence intensity of a round area (with a $5 \mu \mathrm{m}$ radius), roughly corresponding to half the nuclear area, was measured.

\subsection{Evaluation of the effects on cell proliferation}

The effects of SPD addition were also evaluated on cell growth. To this end, 8 primary chondrocyte cultures were established from 8 dif(as assessed through the 8-oxo-dG staining) were evaluated by confocal 
ferent patients and their differential proliferative abilities were assessed as described in Ref. [41] by means of the PicoGreen dsDNA (Molecular Probes, Eugene, OR) quantitation reagent. 1000 cells were plated in triplicate wells of a 96 well plate either in plain medium (10\% FCS DMEM) or with the addition of $100 \mathrm{nM}$ SPD. Cells were left to proliferate and plates were collected at time $0,3,7,10$ and 14 days after plating. At each time the plates were emptied and stored frozen until analysis. $100 \mu \mathrm{l}$ of cell lysis buffer (Molecular Probes) was added to each well and then combined with an equal volume of TE working solution containing the PicoGreen dsDNA quantitation reagent diluted 1:40. After 5 min incubation, the sample fluorescence was measured with a Spectra Max Gemini plate fluorometer (Molecular Devices, Sunnyvale, CA). The instrument was set in the well scan mode with 480 excitation and 540 emission (cut off 515). Values were referred to fluorescence intensity at day 0 and expressed following the formula "percentage of growth increase $=\left(\right.$ fluorescence $t_{n}$-fluorescence $\left.t_{0}\right) /$ fluorescence $t_{0} * 100$ ".

\subsection{Statistics}

The graphs represent the cumulative analysis of at least four different experiments or otherwise stated (in the Figure legend). All the data presented in graphs are expressed as means \pm SEM. Means of groups were compared with GraphPad Prism 5 statistical software (GraphPad Software, Inc.) and analyzed for statistical significance $(*=p<0.05$, $* *=p<0.01, \cdots * *=p<0.001$ ). Differences were considered statistically significant at $\mathrm{p}<0.05$. Comparison of different groups of samples was performed by mean of the Student's t-test, either for paired or unpaired samples, where appropriate.

\section{Results and discussion}

\subsection{SPD reduces hydrogen peroxide cytotoxicity and OS markers}

In order to investigate the chondroprotective effects of SPD against OS, chondrocytes were pretreated with $100 \mathrm{nM}$ SPD for $24 \mathrm{~h}$ before the addition of $\mathrm{H}_{2} \mathrm{O}_{2}$. Then the extent of cell death and oxidative DNA damage was evaluated (Fig. 1).

SPD pre-treatment was able to attenuate cell death after $\mathrm{H}_{2} \mathrm{O}_{2}$ exposure in a statistically significant manner (Fig. $1 \mathrm{~A}$ ).

Accordingly, SPD afforded protection against DNA damage. The chondroprotective effects exerted by SPD were also evident as a significantly reduced $\gamma \mathrm{H} 2 \mathrm{AX}$ signal, a marker of double strand breaks (Fig. 1B). Indeed the graph shows that $\mathrm{H}_{2} \mathrm{O}_{2}$ strongly induces the augmentation of $\gamma \mathrm{H} 2 \mathrm{AX}$ foci due to OS, but SPD pre-treatment reverts this phenotype.

$\gamma \mathrm{H} 2 \mathrm{AX}$ foci are marker of double strand breaks (DSBs) that occur after exposure to OS in order to organize the DNA damage response and repair [42]. Phosphorylation of H2AX is one of the earlier cellular response to DSBs that accumulates in DNA foci and activates complex molecular pathways [43] collectively known as the DNA damage response (DDR) [44] to obtain rapid and efficient error correction and to preserve genomic stability.

8-oxo-dG is a known marker of oxidative damage to DNA [45,46] that in chondrocytes mostly stains mitochondrial DNA [47], more susceptible to oxidative damage and less protected compared to genomic DNA [48]. Furthermore, 8-oxo-dG staining can also indicate oxidative damage to RNA that severely hampers RNA synthesis and protein translation [49]. In fact, $\mathrm{H}_{2} \mathrm{O}_{2}$ led to a strong increase of 8-oxo-dG signal, while SPD pre-treatment was able to reduce the intensity of 8-oxo-dG staining in $\mathrm{H}_{2} \mathrm{O}_{2}$ treated cells (Fig. $1 \mathrm{~B}$, first row of images and right graph). Moreover, the subcellular distribution of this signal markedly changed, with less signal in the nuclear area.

To gain insight in the modality of the cytoprotective action of SPD, caspase 3 (CP3) activation was investigated. Caspase 3-like activity was induced by hydrogen peroxide, and significantly reduced by SPD in both unstimulated and $\mathrm{H}_{2} \mathrm{O}_{2}$-stimulated conditions (Fig. 1B, penultimate right graph). Accordingly, the immunofluorescent pattern of active CP3 changed from an intense nuclear dotted pattern $\left(\mathrm{H}_{2} \mathrm{O}_{2}\right)$ to a more diffuse and more mitochondrial pattern (SPD $+\mathrm{H}_{2} \mathrm{O}_{2}$ ), similar to that of cells treated with SPD only (high magnification in Fig. 1B). To provide a quantitative assessment of this intracellular re-localization, the nuclear/cytoplasmic ratios of the signal were measured as described above, and presented in the lower right graph. The findings of differential localization of active $\mathrm{CP} 3$ and particularly its preferential localization in the nucleus after $\mathrm{H}_{2} \mathrm{O}_{2}$ delivery is in keeping with information at https://www.proteinatlas.org/ENSG00000164305-CASP3/ cell\#human [50]. Localization of CP3 in the nucleoplasm and in the mitochondria corresponds to a unique distribution pattern among the caspases involved in the crosstalk between DNA damage and apoptosis, that is consistent with an activation in the mitochondria of the enzyme that then moves to the nuclei to activate endonucleases that execute DNA double strand breaks $[37,51]$.

This possibly indicates that also in chondrocytes SPD is effective in counteracting CP3 activity as previously reported in nervous cells [52], thus preventing endonuclease activation [37]. At the same time SPD may sustain a mitochondrial activity of caspase 3, recently put in connection with mitochondrial biogenesis [53].

\subsection{SPD chondroprotective action is mediated by induction of autophagy and mitophagy}

In their previous work Yang et al. [52] indicated that the protective effects of SPD in neurons occurs through autophagy, namely through induction of Beclin-1 (BECN-1), and that SPD is able to exert a CP3 inhibiting activity, thus sparing BECN-1 from CP3-mediated cleavage. Noteworthy, these authors also showed that protective effects are lost upon $B E C N-1$ silencing. In order to ascertain the involvement of autophagy in the chondroprotective action of SPD, we first assessed the expression of the autophagy-related proteins BECN-1 (initiator of phagophore), LC3-II (marker of autophagosome) and p62 (sequestosome 1 protein, required for binding of LC3 and functioning as a selective substrate of autophagy thus serving as a marker of the autophagic flux) in chondrocytes by Western blot.

The amount of BECN-1 was significantly increased by SPD pre-treatment (Fig. 2A). The clear induction of BECN-1 seen in our settings is in keeping with a previous report [11]. The expression of BECN-1 was markedly decreased by $\mathrm{H}_{2} \mathrm{O}_{2}$, suggesting a higher turnover of the protein under OS. However, our Western blot analysis rules out that BECN-1 reduction by $\mathrm{H}_{2} \mathrm{O}_{2}$ is due to caspase-mediated degradation, since no degradation fragments were seen that could be suggestive of either caspase or calpain degradation [54]. Available information suggests an involvement of BECN-1 in a wide array of processes (https:// www.uniprot.org/uniprot/Q14457 [55]), including autophagosome formation and autophagy of the mitochondrion. Therefore, we speculated that BECN-1 reduction may be at least partially due to its degradation in the autophagolysosomes because of the increased autophagic flux when SPD delivery preceded exposure to OS. It is noteworthy that the rate of the autophagic flux impacts on the level of some autophagic proteins, including BECN-1, since they are involved particularly in mitophagy, as previously reported [56]. Other papers have described peculiar patterns for BECN-1, overlapping with mitochondria, endoplasmic reticulum and nuclear laminae [57]. On the other hand, available information indicates that BECN-1 exerts both autophagy-related and unrelated activities but supporting cell health and homeostasis, such as the growth-promoting activity, the apoptosis-inhibiting activity [58] and the DNA damage response [59].

To achieve a deeper insight into the peculiar subcellular distribution of BECN-1 in chondrocytes we carried out some immunofluorescent ex- 
A

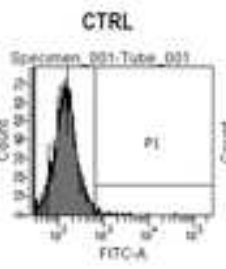

B
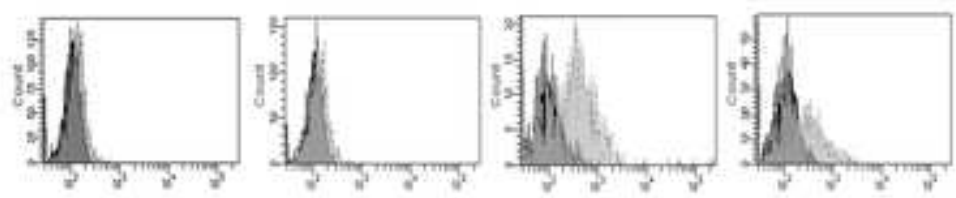

CTRL
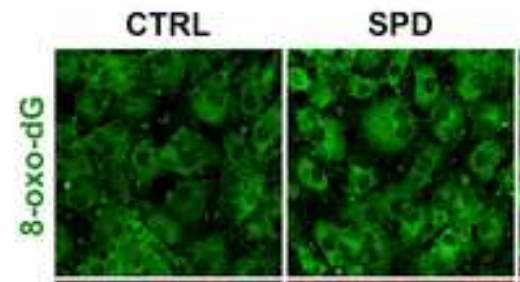

$\mathrm{H}_{2} \mathrm{O}_{2}$
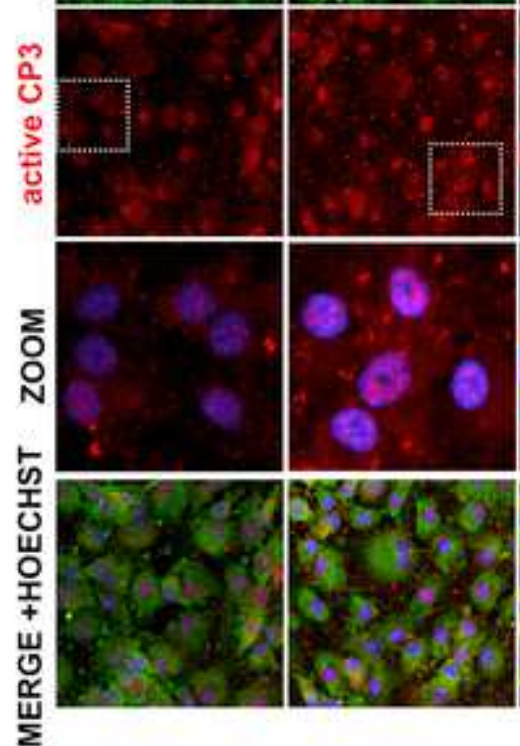

SPo
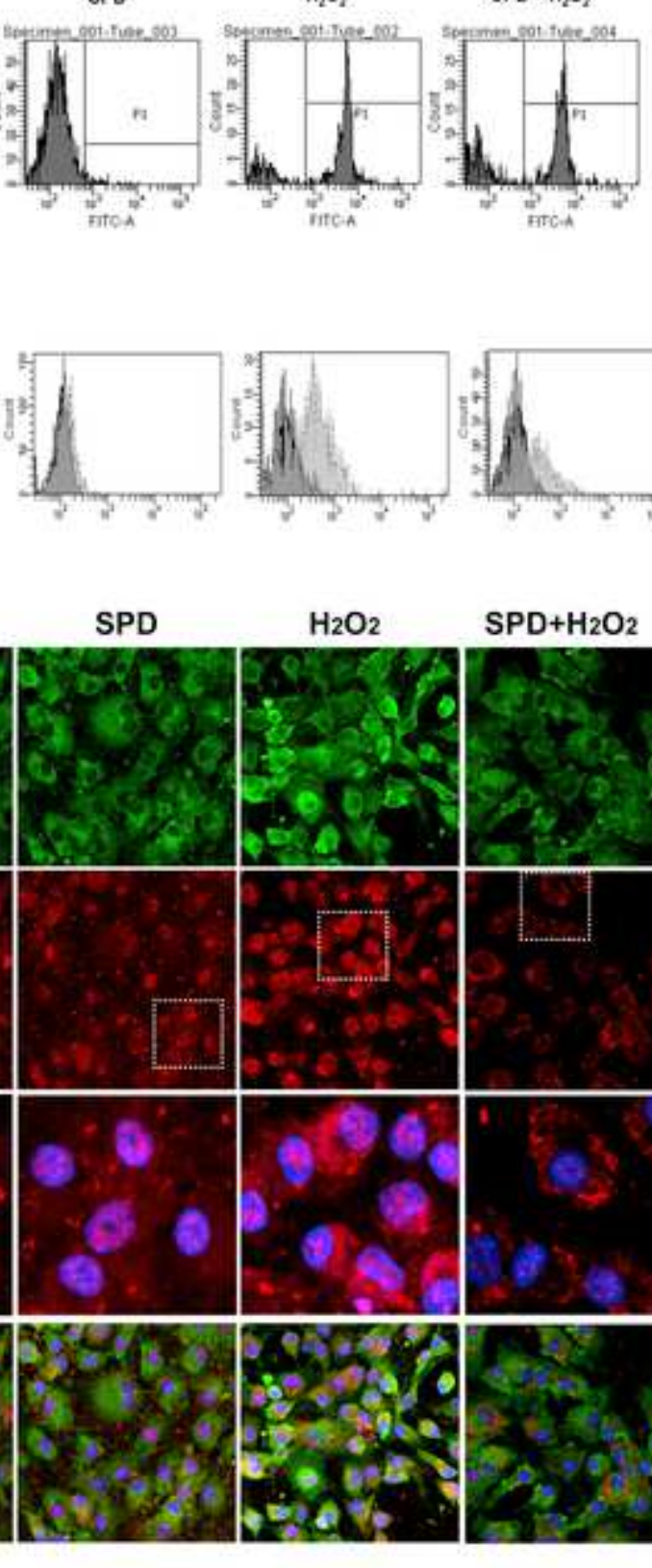

\section{$\mathrm{SPD}+\mathrm{H}_{2} \mathrm{O}_{2}$}
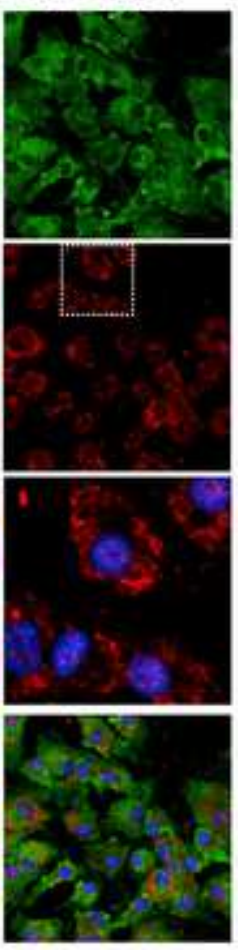
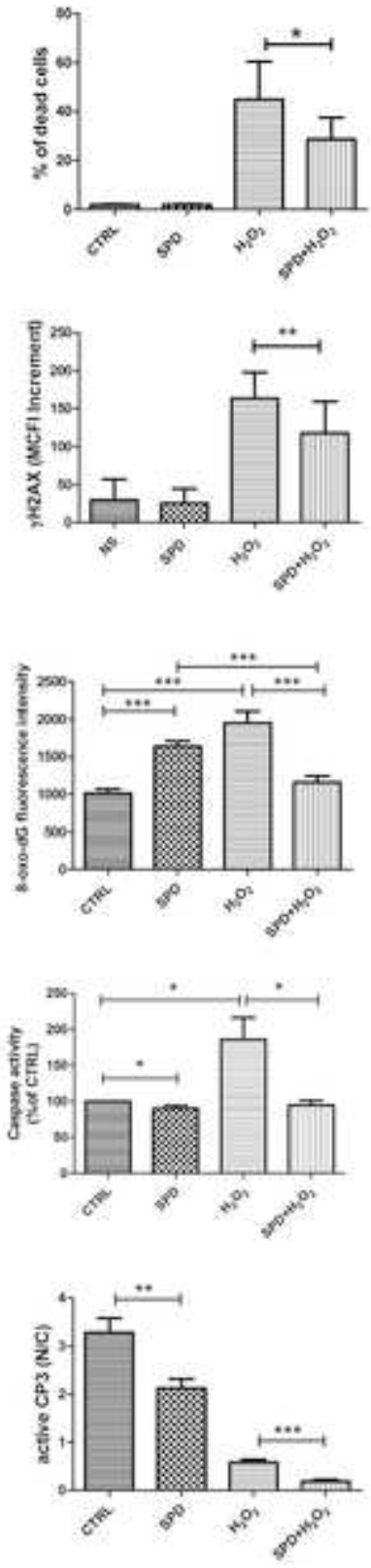

Fig. 1. SPD prevents hydrogen peroxide-induced cell death, DNA damage and caspase 3-activity. (A) SPD pre-treatment significantly reduces cell death, as evidenced by DNA staining (Sytox Green). Left: a representative image of flow cytometry diagram in control (CTRL), spermidine (SPD), hydrogen peroxide $\left(\mathrm{H}_{2} \mathrm{O}_{2}\right)$, spermidine + hydrogen peroxide $\left(\mathrm{SPD}+\mathrm{H}_{2} \mathrm{O}_{2}\right)$ conditions. Right: quantitative histogram graph $(\mathrm{n}=5$, * $\mathrm{p}<0.05)$. (B) SPD pre-treatment significantly reduces DNA damage $(\gamma \mathrm{H} 2 \mathrm{AX}$ and 8-oxo-dG) and active caspase-3 (CP3). Left: a representative image of flow cytometry analysis of the level of $\gamma \mathrm{H} 2 \mathrm{AX}$ in CTRL, SPD, $\mathrm{H}_{2} \mathrm{O}_{2}$ and SPD $+\mathrm{H}_{2} \mathrm{O}_{2}$ conditions. Dark grey histograms: isotype control and light grey histograms: $\gamma \mathrm{H} 2 \mathrm{AX}$-specific antibody. Right graph: cumulative results $\left(\mathrm{n}=5,{ }^{* *} \mathrm{p}<0.01\right)$. The correlated behavior of 8-oxo-dG and active CP3 was investigated by double immunofluorescence and confocal microscopy analysis ( $\mathrm{n}=3$ different experiments). First row: 8-oxo-dG staining (mainly staining mitochondrial DNA) intensity reduced and retained in the cytoplasm in SPD $+\mathrm{H}_{2} \mathrm{O}_{2}$ compared to $\mathrm{H}_{2} \mathrm{O}_{2}$. Right graph: cumulative data of 8-oxo-dG intensity obtained from 20 cells for each condition ( $\mathrm{n}=20$, ***p < 0.001). Second row: active CP3 intensity reduced in SPD $+\mathrm{H}_{2} \mathrm{O}_{2}$ compared to $\mathrm{H}_{2} \mathrm{O}_{2}$ and with a mitochondrial pattern; third row: enlargements from correspondent confocal pictures above, of the insets indicated by the dotted squares; fourth row: merged images from multi-labeled immunofluorescence. Middle right graph: SPD alone or in combination with $\mathrm{H}_{2} \mathrm{O}_{2}$ significantly reduces caspase-3 like activity induced by OS, compared to CTRL and $\mathrm{H}_{2} \mathrm{O}_{2}$, respectively. $\mathrm{CP} 3$ activity was assessed as a function of the accumulation of a fluorescent product following the cleavage of the fluorogenic peptide substrate Ac-Asp-Glu-Val-Asp-7-amido-4- methylcoumarin (Ac-DEVD-AMC). The intensity of the fluorescence signal was then normalized per mg protein, and expressed as fold increase compared to the untreated controls $(\mathrm{n}=4$, *p $<0.05)$; lower right graph: a quantitative analysis of the nuclear-cytoplasm ratio (N/C) shows an increased cytosolic localization of active CP3 in SPD and SPD $+\mathrm{H}_{2} \mathrm{O}_{2}$ compared to CTRL and $\mathrm{H}_{2} \mathrm{O}_{2}$, respectively. $(\mathrm{n}=10$, $* * \mathrm{p}<0.01$, ***p $<0.001$ ). (For interpretation of the references to colour in this figure legend, the reader is referred to the Web version of this article.)

periments. In the selected experimental settings, because of "high density" cultures, most chondrocytes are resting, and only about $10 \%$ of them are in the cell cycle. Therefore, at first we focused on BECN-1 imaging in chondrocytes in interphase. In these cells, BECN-1 staining appeared diffuse, with a pattern suggestive of the cytoskeleton and with a stronger staining at the level of the centrosome (microtubule organizing center or MTOC) (Fig. 2A, right image). The centrosome has also relevant functions in controlling many cell processes, including vesicles trafficking and autophagy [60], in keeping with recent findings that point at a loss of metformin protective activities in cells devoid 
A

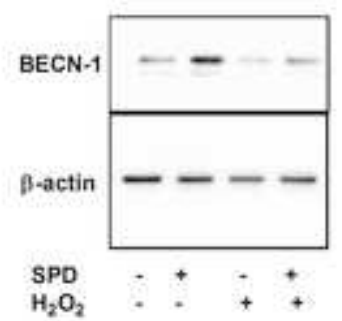

B

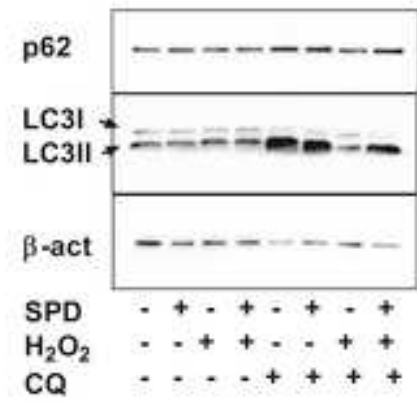

C
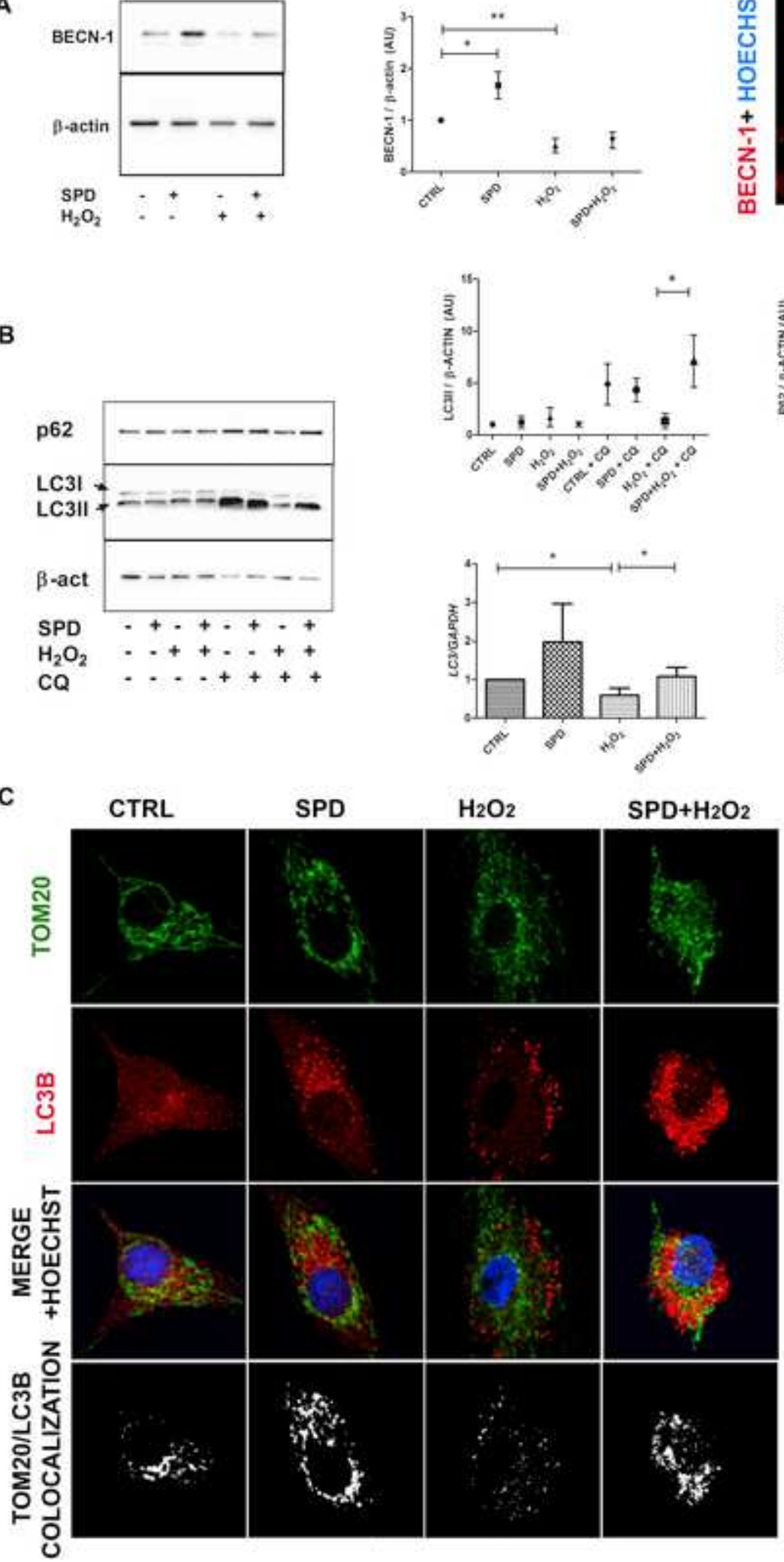

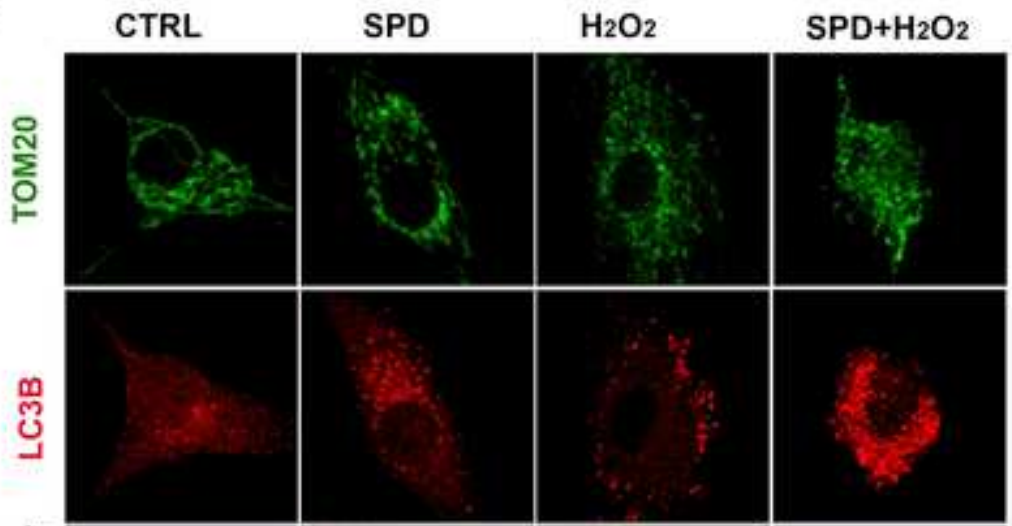

CTRL
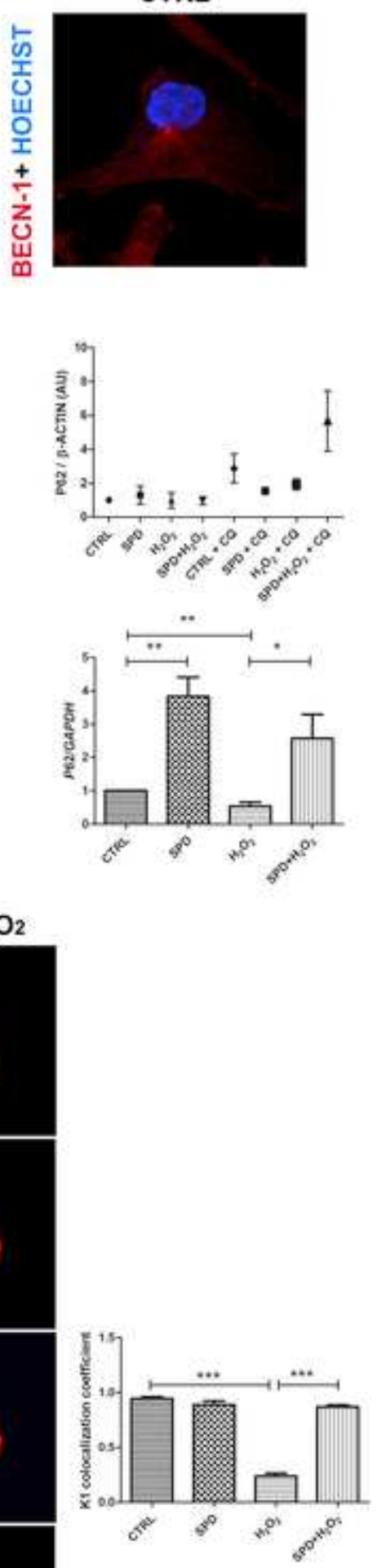

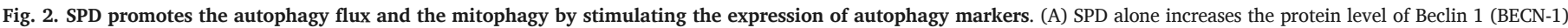

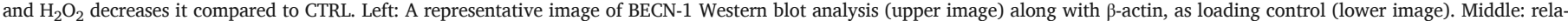

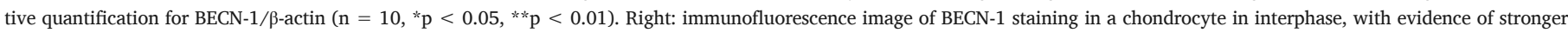

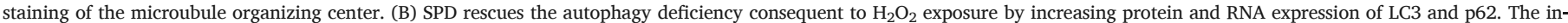




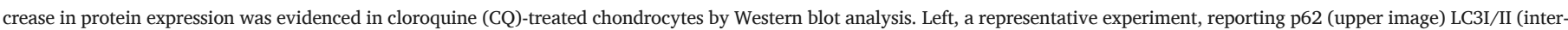

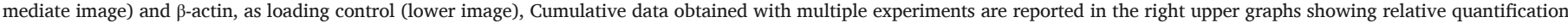

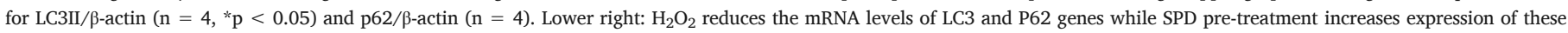

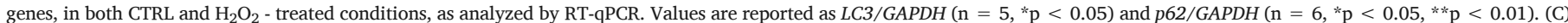

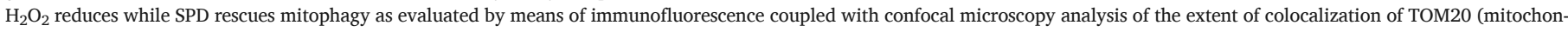

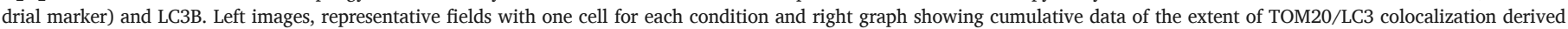
from 15 cells for each condition $(n=15, * * * p<0.001)$.

of ATG6/BECN-1 [61]. All the pleiotropic activities reported for BECN-1 are in keeping with its wide intracellular distribution in chondrocytes and its connections with the MTOC and the cytoskeleton that also suggest a pivotal role in cell division, in accordance with previous literature [62]. Indeed, in both the CTRL and SPD treatment conditions, figures of dividing cells were appreciated where a much stronger BECN-1 staining depicts each mitotic phase, as shown in Fig. 5A. On the other hand, these figures were completely absent in hydrogen peroxide treated cells, suggesting OS direct damaging effect on BECN-1 that could be more accessible in dividing cells. Furthermore, in the magnification shown in Fig. 5A, colocalization of red (BECN-1) and green (LC3B) signals suggests a functional connection of these two autophagy related proteins in cells undergoing mitosis (https://www.uniprot.org/uniprot/Q14457 [55]).

Western blot LC3 analysis suggested that our experimental settings, and possibly the starvation step [4] greatly enhanced autophagy, as indicated by a much higher intensity of the LC3-II versus the LC3-I band in all conditions.

Following published guidelines [13], for a better assessment of the autophagy flux we evaluated the expression of autophagy related proteins of the cells in all four conditions comparing the pattern obtained from cells either untreated or treated with CQ, a chemical inhibitor of autophagosome-lysosome fusion and thus of the late steps of autophagy. The CQ block of the autophagic flux allowed accumulation of autophagosomes and LC3-II and therefore also to appreciate across the different conditions the differential levels of the autophagy-related proteins, that otherwise appear to be expressed at a homogeneous level. Therefore, in CQ-treated cells, we observed a significantly increased expression of the autophagosomal marker LC3-II when SPD pre-treatment preceded $\mathrm{H}_{2} \mathrm{O}_{2}$ exposure (Fig. 2B, left image and upper left graph).

The analysis of p62 showed a similar trend, yet failing to reach statistical significance. Collectively, these data suggest that both autophagy and autophagic flux are enhanced when SPD pre-treatment precedes exposure to OS and that the failure to appreciate these effects without CQ is due to a high level of autophagy associated with the experimental setting of hyperconfluent cells (Fig. 2B, right upper panels). The autophagy-promoting activity of SPD particularly after exposure to $\mathrm{H}_{2} \mathrm{O}_{2}$ was in keeping with the enhanced transcription of both LC3 and p62 genes, that further points at their increased turnover due to an enhanced autophagic flux. Conversely, exposure to $\mathrm{H}_{2} \mathrm{O}_{2}$ only, reduced their transcription compared to the CTRL condition (Fig. 2B, right lower graphs).

The complex regulation of autophagy by redox signaling and their crosstalk has been discussed in detail elsewhere [63]. Interestingly, starvation itself as well as caloric restriction mimetics lead to intracellular ROS accumulation, that in turn promotes induction of autophagy. Conversely, exogenous $\mathrm{H}_{2} \mathrm{O}_{2}$ delivery has been reported to impair the autophagic flux, through lysosomal dysfunction that underlies senescence induction [64]. We here report that delivery of $\mathrm{H}_{2} \mathrm{O}_{2}$ in chondrocytes has also a direct damaging effect on BECN-1, and reduced transcription of LC3 and p62, thus possibly contributing to the impairment of the autophagic flux.

Analysis of the type of autophagy occurring in chondrocytes using the immunofluorescent co-localization of the signals of TOM20 (green, a mitochondrial marker) and LC3B (red, a marker of autophagolysosome) showed a strong reduction of mitophagy after $\mathrm{H}_{2} \mathrm{O}_{2}$ but also the ability of SPD to rescue mitochondrial sequestering into the autophagosomes (orange, mitophagy) in all conditions. Reconstruction of one rep- resentative cell for each condition was undertaken and shown in Fig. 2C. These images indicate that in the SPD $+\mathrm{H}_{2} \mathrm{O}_{2}$ condition, the areas of stronger LC3B signals co-localized with TOM20 antigen, thus indicating mitophagy. Moreover, a quantitative comparison of the intensity of colocalized pixels (filtering the events with fluorescence intensity close to the bisector that were rendered in white in lower images of Fig. 2C) showed a significantly higher co-localized signal of TOM20 and LC3B when SPD pre-treatment preceded $\mathrm{H}_{2} \mathrm{O}_{2}$ delivery (Fig. 2C, right graph).

The paired evaluation of the oxidative markers $(\gamma \mathrm{H} 2 \mathrm{AX}$ and 8-oxo-dG, Fig. 1) indicated that SPD pre-treatment allowed an enhanced turnover of damaged organelles.

Facilitation of mitophagy has recently put in connection with prevention of inflammation [65].

\subsection{SPD chondroprotective action is lost upon blockage of autophagy}

To fully confirm the role of autophagy in the chondroprotection mediated by SPD, a set of experiments were carried out comparing the viability of cells which had underwent siRNA-mediated silencing of ATG5 (siATG5) versus cells treated with control siRNA (siCTRL). The efficiency of the knockdown was investigated by Western blot (Fig. 3A, left image). Cumulative data derived from 4 experiments showed a reduction approaching $70 \%$ as indicated by the Manufacturer (Fig. 3A, right graph).

ATG5 silencing does not affect chondrocyte viability in control condition, but it does after exposure to OS (Fig. 3B).

Exposure of siATG5 or siCTRL chondrocytes to $\mathrm{H}_{2} \mathrm{O}_{2}$ with or without SPD pre-treatment indicated that the protection afforded by SPD pre-treatment was completely lost in siATG5 chondrocytes that have impaired autophagy, which showed a statistically significant higher cell death compared to siCTRL cells (Fig. 3B).

\subsection{SPD pre-treatment decreases expression of differentiation, catabolic and inflammatory markers in chondrocytes exposed to hydrogen peroxide}

Hydrogen peroxide is a genotoxic reagent and, therefore, chondrocyte exposure to this agent leads to activation of the NF- $\mathrm{kB}$ signaling pathway [3]. NF- $\mathrm{\kappa B}$ has a pivotal role in sustaining osteoarthritis pathogenesis [66], acting at different levels: in determining loss of maturational arrest of chondrocytes in articular cartilage, in promoting release of catabolic enzymes or angiogenetic factors that impact on the homeostasis of this avascular tissue and in increasing expression of NF- $\mathrm{\kappa B}$ target genes such as iNOS and COX2 that contribute to increased release of $\mathrm{NO}$ and prostaglandins, mediators of inflammation and pain.

The analysis carried out in 6 different primary cultures showed that SPD pre-treatment significantly counteracted the hydrogen peroxide-dependent increase of the following mRNAs (Fig. 4A and B): RUNX2 (a transcription factor that is a marker of progressed chondrocyte differentiation [67,68]); MMP13, the pivotal collagen 2 catabolic enzyme; $V E G F$, a key angiogenetic factors and a $\beta$-catenin target gene that previous work has put in connection with the NF- $\mathrm{kB}>\mathrm{MMP}-13>\beta$-catenin axis $[68,69]$. With regards to $S O X 9$, its expression was increased by SPD, in keeping with previous results from our laboratory [33]. Collectively, the increased expression levels of SOX9, RUNX2, MMP13 and $V E G F$ upon delivery of $\mathrm{H}_{2} \mathrm{O}_{2}$ are consistent with a NF- $\mathrm{kB}$ activating activity of the latter [3], and NF- $\mathrm{KB}$ activation in turn exerts a differentiation promoting activity [68]. Despite conflicting literature find- 
A
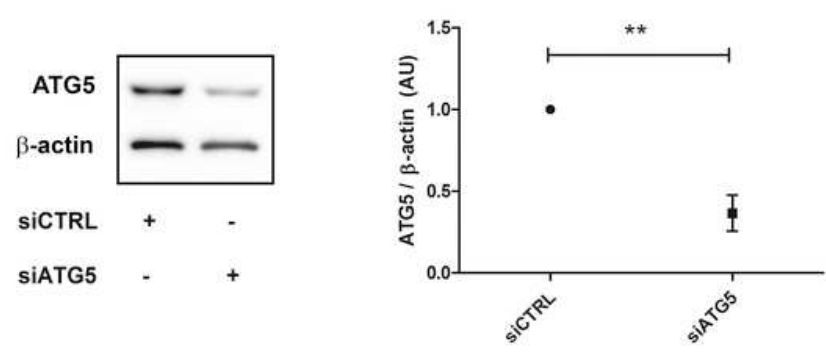

B

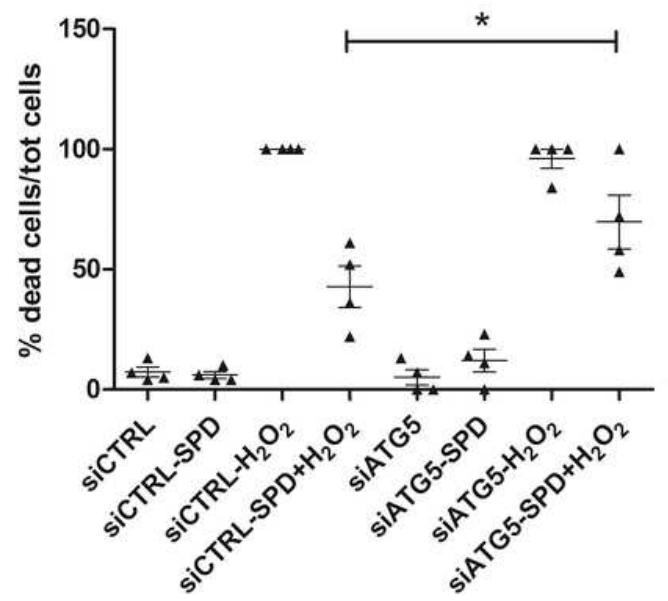

Fig. 3. SPD chondroprotective action is lost upon blockage of autophagy as evident after ATG5 silencing. (A) Primary chondrocytes were transfected for $48 \mathrm{~h}$ with ATG5-siRNA (siATG5) or siRNA negative control (siCTRL). ATG5 levels were determined by western blotting. Left: representative images of the Western blot analysis of one representative example with ATG5 (upper image) along with $\beta$-actin, as loading control (lower image). Right: relative quantification for ATG5/ $\beta$-actin ratio ( $n=4$, **p $<0.01$ ) (B) $24 \mathrm{~h}$ after transfection, siCTRL and siATG5 chondrocytes underwent exposure to SPD and/or $\mathrm{H}_{2} \mathrm{O}_{2}$. At the end of incubation, cells were counted to assess cell viability by trypan blue exclusion test. ( $\mathrm{n}=4$, $\left.{ }^{*} \mathrm{p}<0.05\right)$.

ings [70,71], the SOX9 promoter contains p65 (i.e. NF-кB RelA) binding sites and in mouse limb cartilage RelA has been reported to act as a transcriptional factor for SOX9 induction and chondrogenic differentiation [72].

Moreover, genuine NF- $\kappa$ B targets such as iNOS [73] and COX2 [74] were reduced by SPD pre-treatment both with or without hydrogen peroxide treatment (Fig. 4C), thus suggesting that SPD was able to reduce $\mathrm{NF}-\mathrm{\kappa B}$ activation. These findings are in accordance with previous data pointing at an anti-inflammatory activity of SPD primarily targeted to inhibit NF- $\mathrm{KB}$ activation, through prevention of I $\mathrm{KB} \alpha$ degradation and p65 nuclear translocation [75]. Analysis of p65 nuclear/cytoplasmic localization showed that treatment with CQ (i.e. block of the autophagic flux) was associated with increased nuclear p65 signal (Fig. 4D). At the same time, as expected, the block of the autophagic flux also led to significantly higher 8-oxo-dG signal that possibly further sustains a positive feed-back on NF-kB signaling [3]. These findings confirm that also in chondrocytes, the maintenance of an efficient autophagic flux is required for tuning down NF- $\mathrm{BB}$ activation, and the aging stress response [21].

With regards to OA pathophysiology, our experimental settings could be considered as an in vitro model to predict the higher tolerance to OS of the articular cartilage of people with higher intake of polyamines, in keeping with recent findings that SPD acts as a caloric restriction mimetic, able to improve longevity and homeostasis in several tissues, thus effectively preventing age associated diseases [76]. A recent report compared animal of similar size, i.e. the normal labora- tory mice (mus musculus) with naked mole rats and found that the metabolomic signature of the longevity of the latter is also contributed by higher levels of spermidine related molecules [8]. Future work could address in vivo the OA attenuating activity of SPD, either delivered as a dietary supplement or intra-articularly injected or topically applied in a DMM (destabilization of medial meniscus) model [77]. The DMM is at present the model of choice to test the effectiveness of new potential OA treatments and depending upon the schedule of delivery (i.e. at the time of surgery or at the stage of mild-to-moderate or moderate-to-severe $\mathrm{OA}$ ), it lends itself to compare both the preventive and the therapeutic activity [78]. At the same time these future in vivo studies could allow to estimate the intra-articular bioavailability of spermidine as well as other pharmacokinetic aspects. 3.5. SPD treatment enhances cell proliferation of chondrocytes.

It is well known that polyamines are essential for cell proliferation [79] and may be speculated that the higher availability of BECN-1 afforded by SPD treatment may favor chondrocyte proliferation.

Evaluation of the cell growth with or without the addition of $100 \mathrm{nM}$ SPD showed its ability to selectively enhance proliferation of cell cultures with low proliferative rates (Fig. 5B). The findings derived from 8 different primary cultures showed that the proliferation curve from day 0 to day 14 led to an increase in the Picogreen signal ranging from 100 to $370 \%$ compared to day 0 . More in detail, in the case of cultures with a normal proliferative behavior SPD addition significantly accelerated cell proliferation at intermediate time points but did not change the final results (Fig. 5B, left graph). On the other hand, in the case of cultures with a low proliferative rate, at early time points SPD significantly increased cell proliferation and its growth promoting activity was maintained until the end of the proliferation curve, leading to an increase of about $100 \%$ compared to the CTRL condition (Fig. 5B, right graph). This suggests that SPD protective activity is also evident in conditions of cell senescence, when cytoprotection may be effectively rescued by an increase in autophagy and removal of damaged organelles or molecules.

\section{Conclusions}

The SPD ability to provide effective protection against the consequences of intracellular OS is confirmed by multiple evidence in our peculiar experimental settings, as outlined in Fig. 6: against both cell and DNA damage (CP3 activation, 8-oxo-dG, $\gamma \mathrm{H} 2 \mathrm{AX}$ and cell death) as well as NF- $\mathrm{kB}$ activation and downstream effects on inflammation, ECM remodeling, chondrocyte hypertrophy and angiogenesis.

Among other autophagy related proteins, SPD greatly enhanced BECN-1, a protein with a pivotal role in cell homeostasis through both autophagy related and unrelated activities, and also increased LC3-II and p62 that sustained the enhanced autophagic flux. The loss of SPD-mediated chondroprotection in ATG5-silenced cells confirmed the key role of autophagy in these protective effects.

Taken together, our findings highlight the chondroprotective, anti-oxidant and anti-inflammatory activity of SPD and suggest that the rescue of an efficient autophagic flux is the molecular basis of the protection afforded by SPD against OS. The clearance of damaged organelles and molecules is an effective mean to counteract cell senescence that has a pivotal role in OA pathogenesis and in this perspective we believe that the findings obtained in intracellular oxidative damage, inflammation and proliferation argue in favor of an effective SPD anti-senescence activity.

Considering the importance of non-invasive strategies in the treatment of OA, we propose SPD as a promising candidate for a non-pharmacologic treatment of OA.

\section{Funding sources}

This research was funded by Ministero dell'Istruzione, dell'Università e della Ricerca, Italy (FIRB Grant: RBAP10KCNS); Ministero 
A

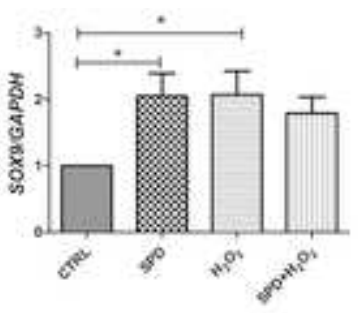

B

C
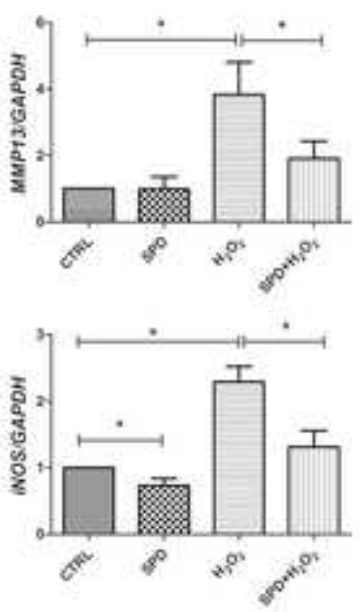

D
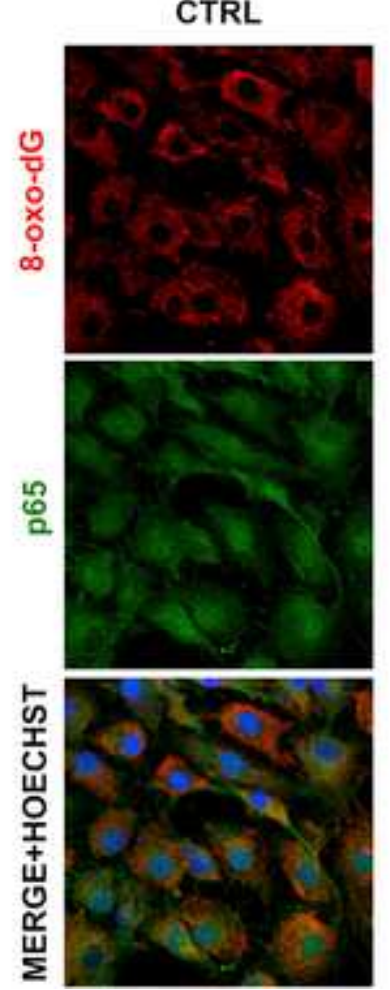
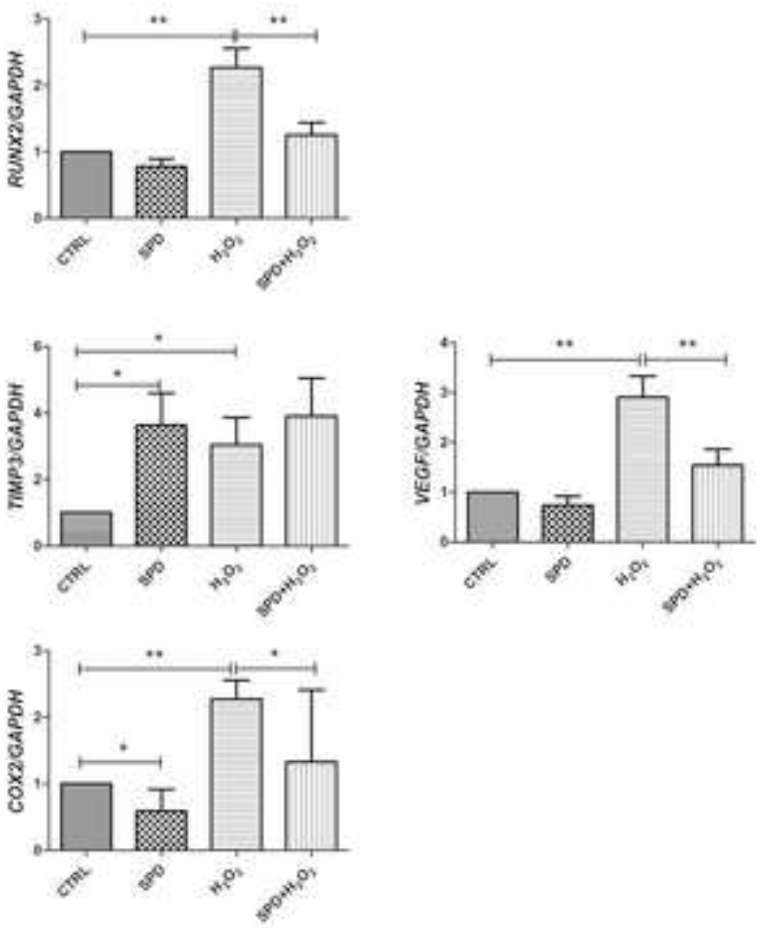
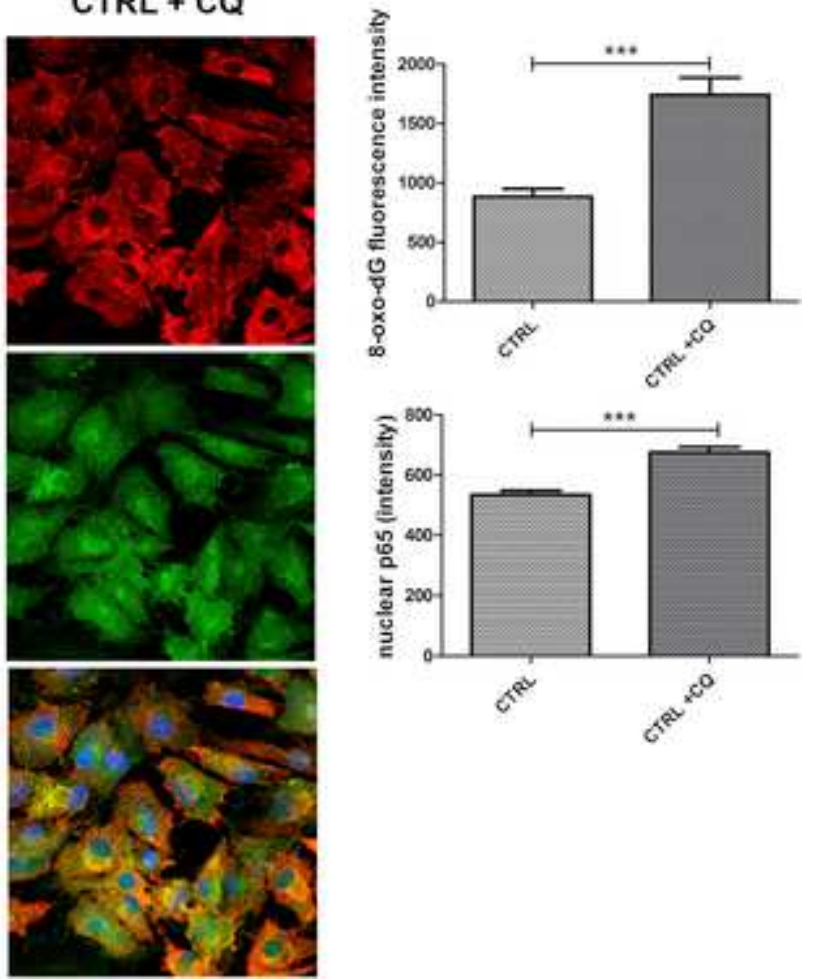

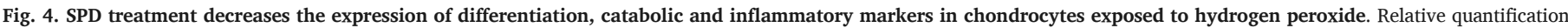

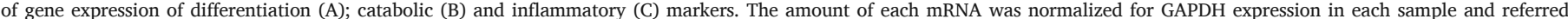

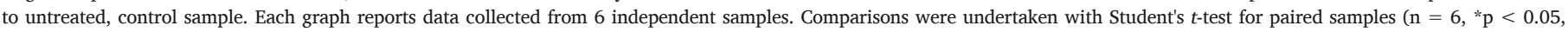

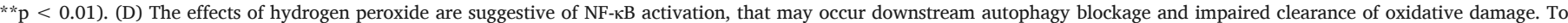

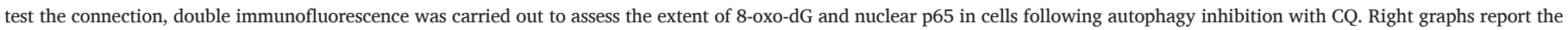
comparisons of the intensity of 8-oxo-dG staining $\left(\mathrm{n}=20,{ }^{* *} \mathrm{p}<0.001\right)$ and of nuclear $\mathrm{p} 65\left(\mathrm{n}=10\right.$, $\left.{ }^{* *} \mathrm{p}<0.001\right)$ between cells treated with or without CQ. 
A
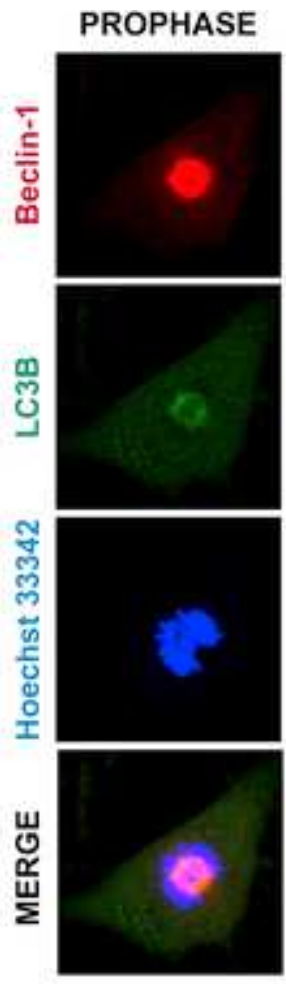
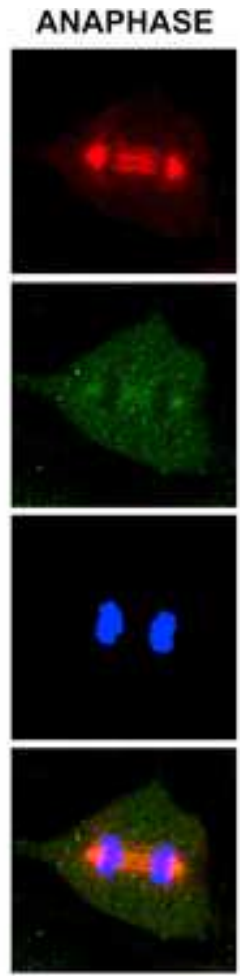
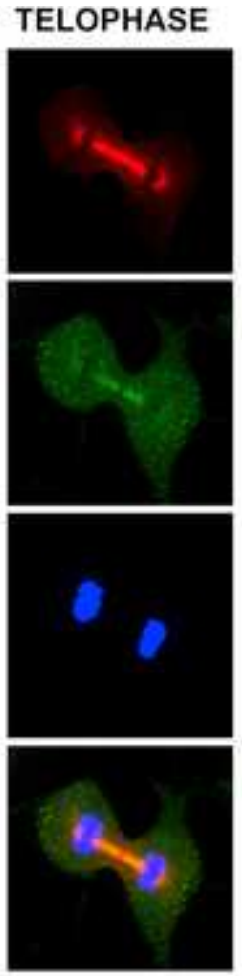
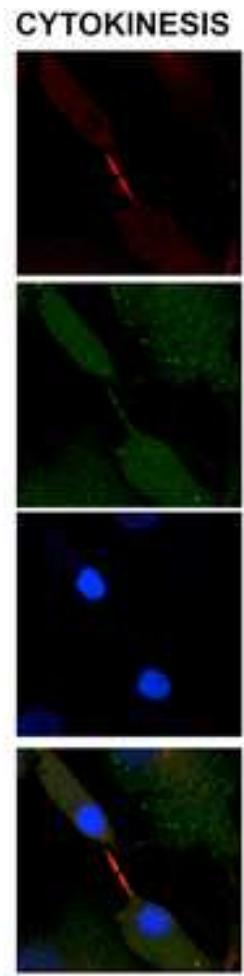
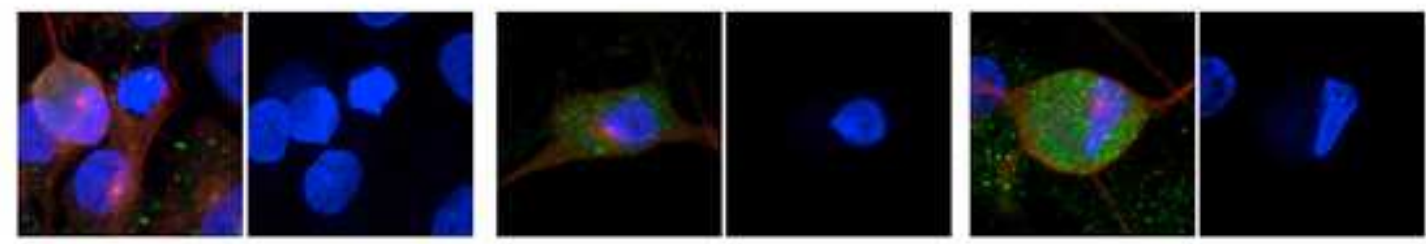

B
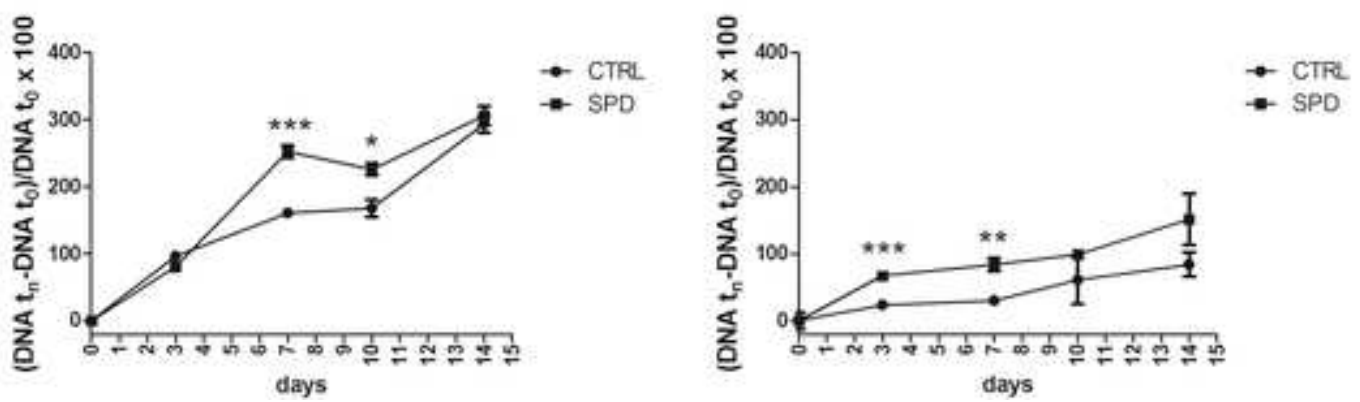

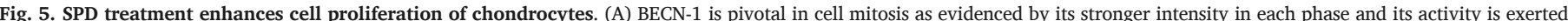

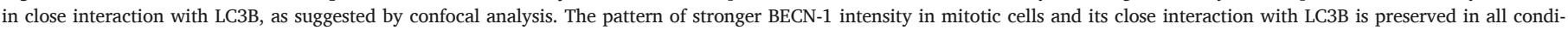

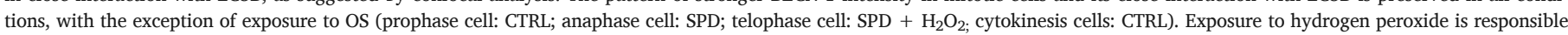

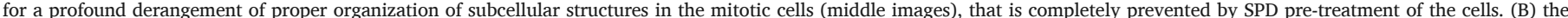

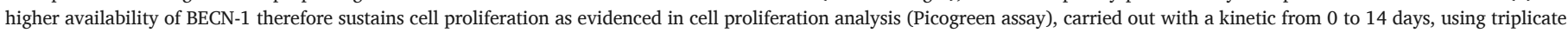

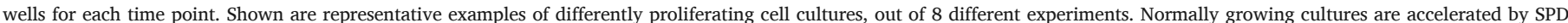

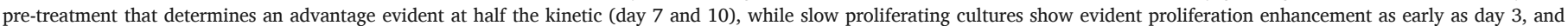
maintain an advantage till the end of the culture, with increased cell recovery in SPD treated samples. ( $\mathrm{n}=3,{ }^{*} \mathrm{p}<0.05$, ${ }^{* *} \mathrm{p}<0.01$, ${ }^{* * *} \mathrm{p}<0.001$ ). 


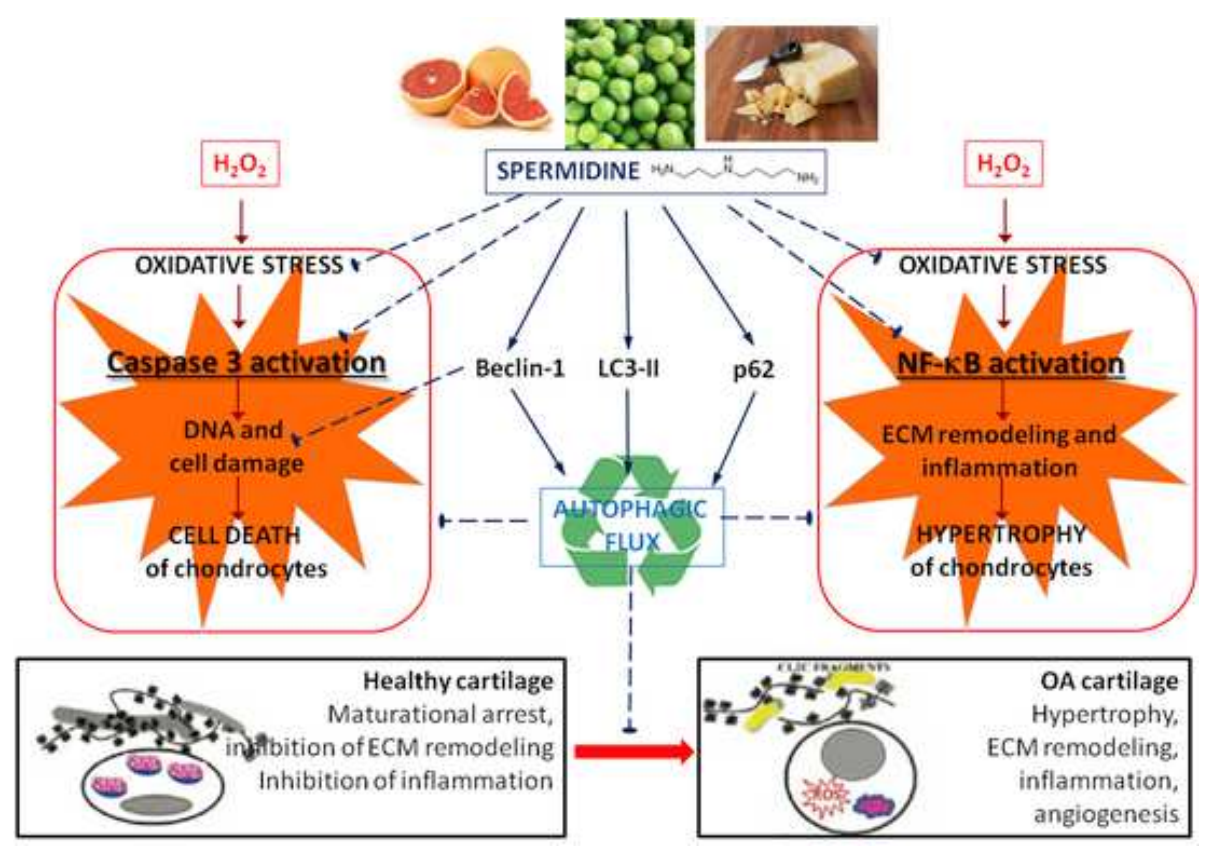

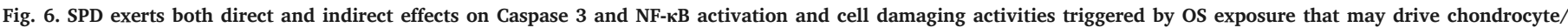

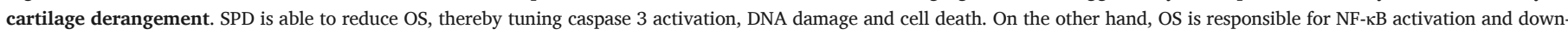

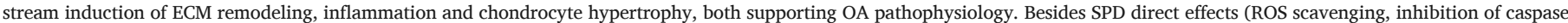

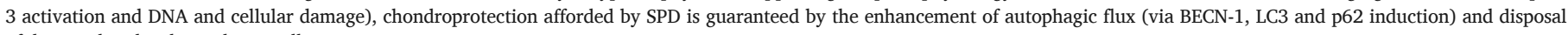
of damaged molecules and organelles.

della Salute, Italy (Fondi Cinque per Mille), University of Bologna (RFO) and Fondazione del Monte di Bologna e Ravenna (Grant: Spermidine as "disease modifying drug" in osteoarthritis: evidence of homeostatic effects in articular chondrocytes).

The funding sources had no involvement in the study design, in the collection, analysis and interpretation of the data, in the writing of the report and in the decision to submit the article.

\section{Declaration of competing interest}

Authors do not have any conflict of interest to declare.

\section{Appendix A. Supplementary data}

Supplementary data to this article can be found online at

\section{References}

[1] M Cross, E Smith, D Hoy, S Nolte, I Ackerman, M Fransen, L Bridgett, S Williams, F Guillemin, C L Hill, L L Laslett, G Jones, F Cicuttini, R Osborne, T Vos, R Buchbinder, A Woolf, L March, The global burden of hip and knee osteoarthritis: estimates from the global burden of disease 2010 study, Ann. Rheum. Dis. 73 (7) (2014) 1323-1330.

[2] M Minguzzi, S Cetrullo, S D’Adamo, Y Silvestri, F Flamigni, R M Borzi, Emerging players at the intersection of chondrocyte loss of maturational arrest, oxidative stress, senescence and low-grade inflammation in osteoarthritis, Oxid Med Cell Longev (2018) 30752932018

[3] A Oeckinghaus, S Ghosh, The NF-kappaB family of transcription factors and its regulation, Cold Spring Harb Perspect Biol 1 (4) (2009) a000034.

[4] M K Lotz, B Carames, Autophagy and cartilage homeostasis mechanisms in joint health, aging and OA, Nat. Rev. Rheumatol. 7 (10) (2011) 579-587.

[5] A Goutas, C Syrrou, I Papathanasiou, A Tsezou, V Trachana, The autophagic response to oxidative stress in osteoarthritic chondrocytes is deregulated, Free Radic. Biol. Med. 126 (2018) 122-132.

[6] T Eisenberg, H Knauer, A Schauer, S Buttner, C Ruckenstuhl, D Carmona-Gutierrez, J Ring, S Schroeder, C Magnes, L Antonacci, H Fussi, L Deszcz, R Hartl, E Schraml, A Criollo, E Megalou, D Weiskopf, P Laun, G Heeren, M Breitenbach, B Grubeck-Loebenstein, E Herker, B Fahrenkrog, K U Frohlich, F Sinner, N Tavernarakis, N Minois, G Kroemer, F Madeo, Induction of autophagy by spermidine promotes longevity, Nat. Cell Biol. 11 (11) (2009) 1305-1314.
[7] F Madeo, M A Bauer, D Carmona-Gutierrez, G Kroemer, Spermidine: a physiological autophagy inducer acting as an anti-aging vitamin in humans?, Autophagy 15 (1) (2019) 165-168.

[8] M Viltard, S Durand, M Perez-Lanzon, F Aprahamian, D Lefevre, C Leroy, F Madeo, G Kroemer, G Friedlander, The metabolomic signature of extreme longevity: naked mole rats versus mice, Aging (N Y) 11 (14) (2019) 4783-4800.

[9] Z Zheng, Z G Wang, Y Chen, J Chen, S Khor, J Li, Z He, Q Wang, H Zhang, K Xu, G Fanghua, J Xiao, X Wang, Spermidine promotes nucleus pulposus autophagy as a protective mechanism against apoptosis and ameliorates disc degeneration, J. Cell Mol. Med. 22 (6) (2018) 3086-3096.

[10] J Yan, J Y Yan, Y X Wang, Y N Ling, X D Song, S Y Wang, H O Liu, O C Liu, Y Zhang, P Z Yang, X B Wang, A H Chen, Spermidine-enhanced autophagic flux improves cardiac dysfunction following myocardial infarction by targeting the AMPK/mTOR signalling pathway, Br. J. Pharmacol. 176 (17) (2019) 3126-3142.

[11] P K Sacitharan, S Lwin, G B Gharios, J R Edwards, Spermidine restores dysregulated autophagy and polyamine synthesis in aged and osteoarthritic chondrocytes via EP300, Exp. Mol. Med. 50 (9) (2018) 123.

[12] R M Borzi, S Cetrullo, S D'Adamo, M Minguzzi, F Flamigni, Spermidine restores dysregulated autophagy and polyamine synthesis in aged and osteoarthritic chondrocytes via EP300, Exp. Mol. Med. 51 (3) (2019) 25.

[13] N Mizushima, T Yoshimori, How to interpret LC3 immunoblotting, Autophagy 3 (6) (2007) 542-545

[14] M Otero, M Favero, C Dragomir, K E Hachem, K Hashimoto, D A Plumb, M B Goldring, Human chondrocyte cultures as models of cartilage-specific gene regulation, Methods Mol. Biol. 806 (2012) 301-336.

[15] Y Henrotin, B Kurz, T Aigner, Oxygen and reactive oxygen species in cartilage degradation: friends or foes?, Osteoarthritis Cartilage 13 (8) (2005) 643-654.

[16] E S Helton, X Chen, p53 modulation of the DNA damage response, J. Cell. Biochem. 100 (4) (2007) 883-896.

[17] I Han, H J Park, S C Seong, S Lee, I G Kim, M C Lee, Role of transglutaminase 2 in apoptosis induced by hydrogen peroxide in human chondrocytes, J. Orthop. Res. 29 (2) (2011) 252-257.

[18] N Sun, R J Youle, T Finkel, The mitochondrial basis of aging, Mol. Cell 61 (5) (2016) 654-666

[19] L Habiballa, H Salmonowicz, J F Passos, Mitochondria and cellular senescence: implications for musculoskeletal ageing, Free Radic. Biol. Med. 132 (2019) 3-10.

[20] R Liu-Bryan, R Terkeltaub, Emerging regulators of the inflammatory process in osteoarthritis, Nat. Rev. Rheumatol. 11 (1) (2015) 35-44.

[21] M C Haigis, B A Yankner, The aging stress response, Mol. Cell 40 (2) (2010) 333-344.

[22] F d'Adda di Fagagna, Living on a break: cellular senescence as a DNA-damage response, Nat. Rev. Canc. 8 (7) (2008) 512-522.

[23] A Freund, A V Orjalo, P Y Desprez, J Campisi, Inflammatory networks during cellular senescence: causes and consequences, Trends Mol. Med. 16 (5) (2010) 238-246.

[24] O H Jeon, N David, J Campisi, J H Elisseeff, Senescent cells and osteoarthritis: a painful connection, J. Clin. Invest. 128 (4) (2018) 1229-1237.

[25] F Madeo, T Eisenberg, F Pietrocola, G Kroemer, Spermidine in health and disease, Science 359 (6374) (2018). 
[26] R F Loeser, J A Collins, B O Diekman, Ageing and the pathogenesis of osteoarthritis, Nat. Rev. Rheumatol. 12 (7) (2016) 412-420.

[27] C Vinatier, E Dominguez, J Guicheux, B Carames, Role of the inflammation-autophagy-senescence integrative network in osteoarthritis, Front. Physiol. 9 (2018) 706.

[28] C Kang, S J Elledge, How autophagy both activates and inhibits cellular senescence, Autophagy 12 (5) (2016) 898-899.

[29] Y Kwon, J W Kim, J A Jeoung, M S Kim, C Kang, Autophagy is pro-senescence when seen in close-up, but anti-senescence in long-shot, Mol. Cell. 40 (9) (2017) 607-612.

[30] S Guidotti, M Minguzzi, D Platano, L Cattini, G Trisolino, E Mariani, R M Borzi, Lithium chloride dependent glycogen synthase kinase 3 inactivation links oxidative DNA damage, hypertrophy and senescence in human articular chondrocytes and reproduces chondrocyte phenotype of obese osteoarthritis patients, PloS One 10 (11) (2015) e0143865.

[31] L Luo, T Cruz, C McCulloch, Interleukin 1-induced calcium signalling in chondrocytes requires focal adhesions, Biochem. J. 324 (Pt 2) (1997) 653-658.

[32] C A Ghisalberti, R M Borzi, S Cetrullo, F Flamigni, G Cairo, Soft TCPTP agonism-novel target to rescue airway epithelial integrity by exogenous spermidine, Front. Pharmacol. 7 (2016) 147.

[33] A Facchini, R M Borzi, E Olivotto, D Platano, S Pagani, S Cetrullo, F Flamigni, Role of polyamines in hypertrophy and terminal differentiation of osteoarthritic chondrocytes, Amino Acids 42 (2-3) (2012) 667-678.

[34] F Vittur, G Lunazzi, L Moro, N Stagni, B de Bernard, M Moretti, G Stanta, F Bacciottini, G Orlandini, N Reali, et al., A possible role for polyamines in cartilage in the mechanism of calcification, Biochim. Biophys. Acta 881 (1) (1986) 38-45.

[35] L Chiaraviglio, J E Kirby, Evaluation of impermeant, DNA-binding dye fluorescence as a real-time readout of eukaryotic cell toxicity in a high throughput screening format, Assay Drug Dev. Technol. 12 (4) (2014) 219-228.

[36] A Facchini, S Cetrullo, S D’Adamo, S Guidotti, M Minguzzi, R M Borzi, F Flamigni, Hydroxytyrosol prevents increase of osteoarthritis markers in human chondrocytes treated with hydrogen peroxide or growth-related oncogene alpha, Plos One 9 (10) (2014) e109724.

[37] S Nowsheen, E S Yang, The intersection between DNA damage response and cell death pathways, Exp. Oncol. 34 (3) (2012) 243-254.

[38] S Cetrullo, S D'Adamo, S Guidotti, R M Borzi, F Flamigni, Hydroxytyrosol prevents chondrocyte death under oxidative stress by inducing autophagy through sirtuin 1-dependent and -independent mechanisms, Biochim. Biophys. Acta 1860 (6) (2016) 1181-1191.

[39] M I Koukourakis, D Kalamida, A Giatromanolaki, C E Zois, E Sivridis, S Pouliliou, A Mitrakas, K C Gatter, A L Harris, Autophagosome proteins LC3A, LC3B and LC3C have distinct subcellular distribution kinetics and expression in cancer cell lines, PloS One 10 (9) (2015) e0137675.

[40] M Riccio, M Dembic, C Cinti, S Santi, Multifluorescence labeling and colocalization analyses, Methods Mol. Biol. 285 (2004) 171-177.

[41] A Facchini, R M Borzi, K B Marcu, C Stefanelli, E Olivotto, M B Goldring, F Flamigni, Polyamine depletion inhibits NF-kappaB binding to DNA and interleukin-8 production in human chondrocytes stimulated by tumor necrosis factor-alpha, J. Cell. Physiol. 204 (3) (2005) 956-963.

[42] N Malaquin, A Carrier-Leclerc, M Dessureault, F Rodier, DDR-mediated crosstalk between DNA-damaged cells and their microenvironment, Front. Genet. 6 (2015) 94.

[43] V Gire, P Roux, D Wynford-Thomas, J M Brondello, V Dulic, DNA damage checkpoint kinase Chk2 triggers replicative senescence, EMBO J. 23 (13) (2004) 2554-2563.

[44] K K Khanna, M F Lavin, S P Jackson, T D Mulhern, ATM, a central controller of cellular responses to DNA damage, Cell Death Differ. 8 (11) (2001) 1052-1065.

[45] E Markkanen, U Hubscher, B van Loon, Regulation of oxidative DNA damage repair: the adenine:8-oxo-guanine problem, Cell Cycle 11 (6) (2012) 1070-1075.

[46] R P Soultanakis, R J Melamede, I A Bespalov, S S Wallace, K B Beckman, B N Ames, D J Taatjes, Y M Janssen-Heininger, Fluorescence detection of 8-oxoguanine in nuclear and mitochondrial DNA of cultured cells using a recombinant Fab and confocal scanning laser microscopy, Free Radic. Biol. Med. 28 (6) (2000) 987-998.

[47] S Guidotti, M Minguzzi, D Platano, S Santi, G Trisolino, G Filardo, E Mariani, R M Borzi, Glycogen synthase kinase-3 beta inhibition links mitochondrial dysfunction, extracellular matrix remodelling and terminal differentiation in chondrocytes, Sci. Rep. 7 (1) (2017) 12059.

[48] G Barja, A Herrero, Oxidative damage to mitochondrial DNA is inversely related to maximum life span in the heart and brain of mammals, Faseb. J. 14 (2) (2000) 312-318.

[49] Z Li, J Wu, C J Deleo, RNA damage and surveillance under oxidative stress, IUBMB Life 58 (10) (2006) 581-588.

[50] Proteinatlas: the human protein atlashttps://www.proteinatlas.org/ ENSG00000164305-CASP3/cell\#human20196 February 2019

[51] X Liu, Y He, F Li, Q Huang, T A Kato, R P Hall, C Y Li, Caspase-3 promotes genetic instability and carcinogenesis, Mol. Cell 58 (2) (2015) 284-296.

[52] Y Yang, S Chen, Y Zhang, X Lin, Y Song, Z Xue, H Qian, S Wang, G Wan, X Zheng, $\mathrm{L}$ Zhang, Induction of autophagy by spermidine is neuroprotective via inhibition of caspase 3-mediated Beclin 1 cleavage, Cell Death Dis. 8 (4) (2017) e2738.

[53] J S Kim, J Y Ha, S J Yang, J H Son, A novel non-apoptotic role of procaspase-3 in the regulation of mitochondrial biogenesis activators, J. Cell. Biochem. 119 (1) (2018) 347-357.
[54] J M Norman, G M Cohen, E T Bampton, The in vitro cleavage of the hAtg proteins by cell death proteases, Autophagy 6 (8) (2010) 1042-1056.

[55] UniProt KB: UniProt knowledgebasehttps://www.uniprot.org/uniprot/ Q14457201917 April 2019

[56] J Nah, S M Yoo, S Jung, E I Jeong, M Park, B K Kaang, Y K Jung, Phosphorylated CAV1 activates autophagy through an interaction with BECN1 under oxidative stress, Cell Death Dis. 8 (5) (2017) e2822.

[57] X H Liang, J Yu, K Brown, B Levine, Beclin 1 contains a leucine-rich nuclear export signal that is required for its autophagy and tumor suppressor function, Canc. Res. 61 (8) (2001) 3443-3449.

[58] Y Cao, D J Klionsky, Physiological functions of Atg6/Beclin 1: a unique autophagy-related protein, Cell Res. 17 (10) (2007) 839-849.

[59] F Xu, Y Fang, L Yan, L Xu, S Zhang, Y Cao, X Zhang, J Xie, G Jiang, C Ge, N An, D Zhou, N Yuan, J Wang, Nuclear localization of Beclin 1 promotes radiation-induced DNA damage repair independent of autophagy, Sci. Rep. 7 (2017) 45385.

[60] B Levine, G Kroemer, Biological functions of autophagy genes: a disease perspective, Cell 176 (1-2) (2019) 11-42.

[61] H J Na, J H Pyo, H J Jeon, J S Park, H Y Chung, M A Yoo, Deficiency of Atg6 impairs beneficial effect of metformin on intestinal stem cell aging in Drosophila, Biochem. Biophys. Res. Commun. 498 (1) (2018) 18-24.

[62] S Fremont, A Gerard, M Galloux, K Janvier, R E Karess, C Berlioz-Torrent, Beclin-1 is required for chromosome congression and proper outer kinetochore assembly, EMBO Rep. 14 (4) (2013) 364-372.

[63] J Lee, S Giordano, J Zhang, Autophagy, mitochondria and oxidative stress: cross-talk and redox signalling, Biochem. J. 441 (2) (2012) 523-540.

[64] H Tai, Z Wang, H Gong, X Han, J Zhou, X Wang, X Wei, Y Ding, N Huang, J Qin, J Zhang, S Wang, F Gao, Z M Chrzanowska-Lightowlers, R Xiang, H Xiao, Autophagy impairment with lysosomal and mitochondrial dysfunction is an important characteristic of oxidative stress-induced senescence, Autophagy 13 (1) (2017) 99-113.

[65] I Gkikas, K Palikaras, $\mathrm{N}$ Tavernarakis, The role of mitophagy in innate immunity, Front. Immunol. 9 (2018) 1283.

[66] K B Marcu, M Otero, E Olivotto, R M Borzi, M B Goldring, NF-kappaB signaling: multiple angles to target OA, Curr. Drug Targets 11 (5) (2010) 599-613.

[67] P M van der Kraan, W B van den Berg, Chondrocyte hypertrophy and osteoarthritis: role in initiation and progression of cartilage degeneration?, Osteoarthritis Cartilage 20 (3) (2012) 223-232.

[68] M B Goldring, M Otero, D A Plumb, C Dragomir, M Favero, K El Hachem, K Hashimoto, H I Roach, E Olivotto, R M Borzi, K B Marcu, Roles of inflammatory and anabolic cytokines in cartilage metabolism: signals and multiple effectors converge upon MMP-13 regulation in osteoarthritis, Eur. Cell. Mater. 21 (2011) 202-220.

[69] R M Borzi, E Olivotto, S Pagani, R Vitellozzi, S Neri, M Battistelli, E Falcieri, A Facchini, F Flamigni, M Penzo, D Platano, S Santi, K B Marcu, Matrix metalloproteinase 13 loss associated with impaired extracellular matrix remodeling disrupts chondrocyte differentiation by concerted effects on multiple regulatory factors, Arthritis Rheum. 62 (8) (2010) 2370-2381.

[70] E Kolettas, H I Muir, J C Barrett, T E Hardingham, Chondrocyte phenotype and cell survival are regulated by culture conditions and by specific cytokines through the expression of Sox-9 transcription factor, Rheumatology 40 (10) (2001) $1146-1156$.

[71] S Murakami, V Lefebvre, B de Crombrugghe, Potent inhibition of the master chondrogenic factor Sox 9 gene by interleukin-1 and tumor necrosis factor-alpha, J. Biol. Chem. 275 (5) (2000) 3687-3692.

[72] M Ushita, T Saito, T Ikeda, F Yano, A Higashikawa, N Ogata, U Chung, K Nakamura, H Kawaguchi, Transcriptional induction of SOX9 by NF-kappaB family member RelA in chondrogenic cells, Osteoarthritis Cartilage 17 (8) (2009) 1065-1075.

[73] K R Morris, R D Lutz, H S Choi, T Kamitani, K Chmura, E D Chan, Role of the NF-kappaB signaling pathway and kappaB cis-regulatory elements on the IRF-1 and iNOS promoter regions in mycobacterial lipoarabinomannan induction of nitric oxide, Infect. Immun. 71 (3) (2003) 1442-1452.

[74] W Et Ackerman, T L Summerfield, D D Vandre, J M Robinson, D A Kniss, Nuclea factor-kappa B regulates inducible prostaglandin E synthase expression in human amnion mesenchymal cells, Biol. Reprod. 78 (1) (2008) 68-76.

75] Y H Choi, H Y Park, Anti-inflammatory effects of spermidine in lipopolysaccharide-stimulated BV2 microglial cells, J. Biomed. Sci. 19 (2012) 31.

[76] F Madeo, D Carmona-Gutierrez, S J Hofer, G Kroemer, Caloric restriction mimetics against age-associated disease: targets, mechanisms, and therapeutic potential, Cell Metabol. 29 (3) (2019) 592-610.

[77] S S Glasson, T J Blanchet, E A Morris, The surgical destabilization of the medial meniscus (DMM) model of osteoarthritis in the 129/SvEv mouse, Osteoarthritis Cartilage 15 (9) (2007) 1061-1069.

[78] Z Zhang, D J Leong, L Xu, Z He, A Wang, M Navati, S J Kim, D M Hirsh, J A Hardin, N J Cobelli, J M Friedman, H B Sun, Curcumin slows osteoarthritis progression and relieves osteoarthritis-associated pain symptoms in a post-traumatic osteoarthritis mouse model, Arthritis Res. Ther. 18 (1) (2016) 128.

[79] A E Pegg, Functions of polyamines in mammals, J. Biol. Chem. 291 (29) (2016) 14904-14912. 